Article

\title{
A Dynamically Adaptable Impedance-Matching System for Midrange Wireless Power Transfer with Misalignment
}

\section{Thuc Phi Duong and Jong-Wook Lee *}

Department of Electronics and Radio Engineering, Kyung Hee University, 1 Sochen, Giheung, Yongin, Gyeonggi 446-701, Korea; E-Mail: thuc@khu.ac.kr

* Author to whom correspondence should be addressed; E-Mail: jwlee@khu.ac.kr;

Tel.: +82-31-201-3730; Fax: +82-31-204-3740.

Academic Editor: K. T. Chau

Received: 8 June 2015 / Accepted: 15 July 2015 / Published: 27 July 2015

\begin{abstract}
To enable the geometrical freedom envisioned for wireless power transfer (WPT), fast dynamic adaptation to unpredictable changes in receiver position is needed. In this paper, we propose an adaptive impedance-searching system that achieves good impedance matching quickly. For fast and robust operation, the proposed method consists of three steps: system calibration, coarse search, and fine search. The proposed WPT system is characterized using distance variation and lateral and angular misalignment between coils. The measured results indicate that the proposed method significantly reduces searching time from a few minutes to approximately one second. Furthermore, the proposed system achieves impedance matching with good accuracy. The robust impedance-searching capability of the proposed system significantly improves power transfer efficiency. At $6.78 \mathrm{MHz}$, we achieve a maximum efficiency of $89.7 \%$ and a high efficiency of $>80 \%$ up to a distance of $50 \mathrm{~cm}$. When the center-to-center misalignment is $35 \mathrm{~cm}$, the efficiency is improved from $48.4 \%$ to $74.1 \%$ with the proposed method. At a distance of $40 \mathrm{~cm}$, the efficiency is higher than $74 \%$ for up to $60^{\circ}$ of angular rotation. These results agree well with the simulated results obtained using a lumped-element circuit model.
\end{abstract}

Keywords: wireless power transmission; resonant coupling; impedance match; dynamic adaptation; efficiency; misalignment 


\section{Introduction}

In recent years, the potential of power transmission through wireless means has attracted a great deal of research and commercial interest [1]. This interest is closely related to the increasing number of mobile devices in our daily lives, which require frequent battery charging because of their high power consumption. To unify the charging protocol of various portable electronic devices, the Wireless Power Consortium (WPC) recently developed a new standard, Qi 1.1 [2]. This standard development reflects the substantial interest in the wide range of wireless power charging applications.

Inductive coupling has been used for wireless power transfer (WPT) in many commercial applications. The drawback of wireless power charging using inductive coupling is that the operation is limited to a very short range. Efforts are being made to extend the operating range, for example, by using repeaters [3] and metamaterials [4,5]. To extend the power transfer to a more convenient midrange distance, WPT based on resonant coupling has recently been demonstrated experimentally [6-10]. This new approach increases the power transmission range by focusing the energy transfer to the narrow frequency region allowed by the high Q-factor. Thus, power transmission is extended to a midrange distance, enabling greater geometrical freedom for mobile device charging.

Nevertheless, the received power level is rather sensitive to both alignment and distance changes between the coils [11]. Any change in coil position from the initial optimal location results in degraded transmission efficiency. The mechanical tuning method, in which the distance and/or angle between the coils are adjusted by physically moving coils, has been used to optimize power efficiency [6]. To restore the original efficiency, manual impedance tuning has been used [12,13]; however, it is time-consuming and requires expertise to obtain the proper matching between coils. In a more automated approach, the frequency tracking technique has been proposed [14]; however, it uses a rather wide frequency band (from $6.17 \mathrm{MHz}$ to $6.78 \mathrm{MHz}$ ), which can potentially cause frequency regulation issues. In [15], the authors reported a WPT system requiring an even larger bandwidth of $14 \mathrm{MHz}$ (from $8 \mathrm{MHz}$ to $22 \mathrm{MHz}$ ). Main consideration in using frequency tracking method is the availability of bandwidth, which complies with frequency regulation not to cause interference with other communicating devices. For power transmission, narrow bandwidth is usually enough. Therefore, impedance matching at a fixed frequency is the preferred approach to avoid interference issue.

Using a fixed frequency of $13.56 \mathrm{MHz}$, adaptive impedance matching employing a capacitor matrix has been studied [16-18]. The adaptation process was performed either manually [16] or automatically using a digital controller [17,18] or mechanical actuator [19].

In these previous works, however, only distance variation was considered; consequently, the results of these experiments cannot be used to evaluate efficiency performance for misaligned coils.

Thus, the previous approaches have drawbacks in practical realization [6,12-15], and moreover, they have limitations in addressing a misaligned receiver [16-19]. Furthermore, several considerations needed for achieving geometric freedom for the midrange WPT have not been studied in detail. These considerations include: (1) system speed in response to coil misalignment; (2) impedance matching accuracy; and (3) efficiency, which depends on coil position, including lateral and angular misaligned cases.

In this paper, we discuss a midrange WPT system that facilitates geometric freedom through fast adaptation capability. To quickly adapt to a dynamically moving coil, impedance matching is performed 
in three steps: system calibration, coarse search, and fine search. Using the coil parameters obtained in the system calibration step, the coarse search step estimates the coil position and determines the initial values for the impedance search. Then, the fine-search step refines the values in the matching network to improve the accuracy. The performance of the proposed WPT system in terms of searching time, matching accuracy, and efficiency is characterized under various practical ranges and positions of the coil. Measurements show that the proposed approach significantly reduces searching time. In addition, high efficiencies are achieved over a wide range of distance and alignment cases. These results show that the proposed approach has good potential to enable the geometrical freedom envisioned for the midrange WPT system.

\section{Design}

\subsection{System Model}

Figure 1a shows the WPT system using resonant coupling. The source voltage is $V_{\mathrm{S}}$, and the source and load resistances are $R_{\mathrm{S}}$ and $R_{\mathrm{L}}$, respectively. The source and load loops are inductively coupled to the multi-turn spiral resonators (Resonators 2 and 3 ). With a large turn ratio, $R_{\mathrm{S}}$ and $R_{\mathrm{L}}$ are converted to a large effective resistance in parallel with the LC resonator [20], creating the high $Q$-factor needed for resonant coupling. The distance between the internal resonators is $d$. The center-to-center distance (lateral misalignment) between the resonators is $\rho$. For the receiver, consisting of resonator 3 and the load loop, there may be angular misalignment $\theta$. The power transmission can be affected by: (1) distance $d$; (2) lateral misalignment $\rho$; (3) angular misalignment $\theta$; and (4) $R_{\mathrm{L}}$ change. In this work, we focus our investigation on achieving high transmission efficiency under the change of geometric parameters (Cases 1-3).

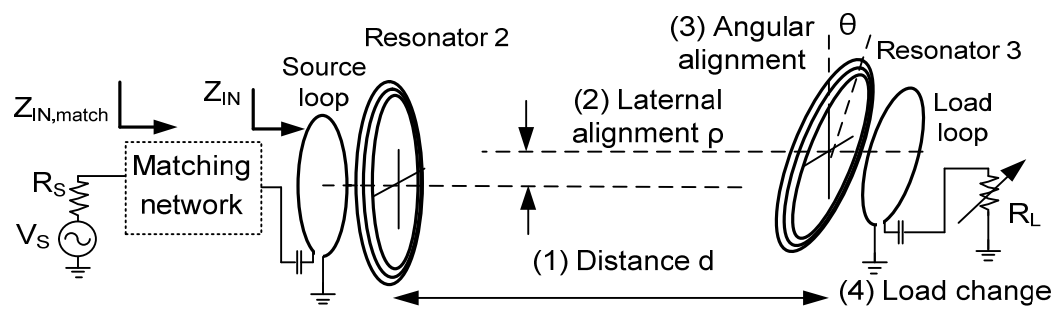

(a)

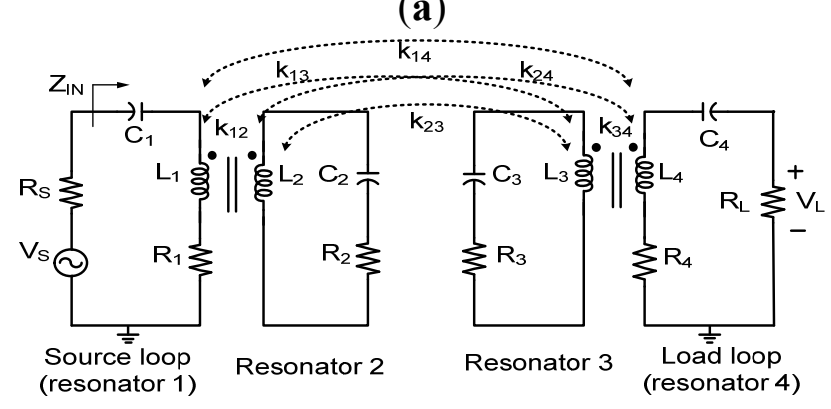

(b)

Figure 1. (a) Schematic of the wireless power transfer (WPT) system using resonant coupling; and (b) equivalent circuit model of resonant coupled coils. 
Because the size of the designed coils is small compared with the wavelength, we build the system model using lumped elements, as shown in Figure 1b. The parameters of each coil are described by lumped RLC elements, where $R_{i}(i=1-4)$ represents ohmic and radiation losses of the coil. The direct-coupling coefficients between each of the two adjacent coils are $k_{12}, k_{23}$, and $k_{34}$. The cross-coupling coefficients are $k_{13}, k_{14}$, and $k_{24}$. Applying basic circuit theory, we obtain the current $I_{i}$ in each coil by using:

$$
\left[\begin{array}{c}
I_{1} \\
I_{2} \\
I_{3} \\
I_{4}
\end{array}\right]=\left[\begin{array}{llll}
Z_{11} & Z_{12} & Z_{13} & Z_{14} \\
Z_{21} & Z_{22} & Z_{23} & Z_{24} \\
Z_{31} & Z_{32} & Z_{33} & Z_{34} \\
Z_{41} & Z_{42} & Z_{43} & Z_{44}
\end{array}\right]^{-1}\left[\begin{array}{c}
V_{\mathrm{S}} \\
0 \\
0 \\
0
\end{array}\right]
$$

where:

$$
\begin{gathered}
Z_{i j}=j \omega M_{i j}=j \omega k_{i j} \sqrt{L_{i} L_{j}}(i, j=1-4, i \neq j) \\
Z_{11}=R_{\mathrm{S}}+R_{1}+j\left(\omega L_{1}-1 / \omega C_{1}\right) \\
Z_{22}=R_{2}+j\left(\omega L_{2}-1 / \omega C_{2}\right) \\
Z_{33}=R_{3}+j\left(\omega L_{3}-1 / \omega C_{3}\right) \\
Z_{44}=R_{L}+R_{4}+j\left(\omega L_{4}-1 / \omega C_{4}\right)
\end{gathered}
$$

At great distance, the strength of the cross-coupling coefficients is substantially smaller than that for direct coupling; we can therefore use the approximation:

$$
Z_{13}=Z_{14}=Z_{24} \cong 0
$$

To simplify the analysis, we assume that all coils are resonating at the same frequency, $\omega_{0}=2 \pi f_{0}$. When $R \mathrm{~s}$ and $R_{\mathrm{L}}$ are connected to the source and load loops, respectively, the $Q$-factors of the loops are affected by these resistors. Then, the impedance values (passive and reciprocal) can be expressed as:

$$
\begin{aligned}
& Z_{12}=j \omega_{0} k_{12} \sqrt{L_{1} L_{2}}=j k_{12} \sqrt{Q_{1} Q_{2}} \sqrt{\left(R_{1}+R_{\mathrm{S}}\right) R_{2}} \\
& Z_{23}=j \omega_{0} k_{23} \sqrt{L_{2} L_{3}}=j k_{23} \sqrt{Q_{2} Q_{3}} \sqrt{R_{2} R_{3}} \\
& Z_{34}=j \omega_{0} k_{34} \sqrt{L_{3} L_{4}}=j k_{34} \sqrt{Q_{3} Q_{4}} \sqrt{R_{3}\left(R_{4}+R_{\mathrm{L}}\right)} \\
& Z_{11}=R_{1}+R_{\mathrm{S}} ; Z_{22}=R_{2} ; Z_{33}=R_{3} ; Z_{44}=R_{4}+R_{\mathrm{L}}
\end{aligned}
$$

where $Q_{i}$ is the quality factor. In the WPT system, the coupling coefficient and $Q$-factor are two key parameters. Solving Equation (1) using the $Z_{i j}$ given in Equation (2), currents $I_{1}$ in the source loop and $I_{4}$ in the load loop are obtained, as shown in Equations (3) and (4):

$$
\begin{aligned}
& I_{1}=\frac{Z_{22} Z_{33} Z_{44}+\left(\omega M_{23}\right)^{2} Z_{44}+\left(\omega M_{34}\right)^{2} Z_{22}}{Z_{11} Z_{22} Z_{33} Z_{44}+\left(\omega M_{12}\right)^{2} Z_{33} Z_{44}+\left(\omega M_{23}\right)^{2} Z_{11} Z_{44}+\left(\omega M_{34}\right)^{2} Z_{11} Z_{22}+\left(\omega M_{12}\right)^{2}\left(\omega M_{34}\right)^{2}} V_{\mathrm{S}} \\
& I_{4}=\frac{j\left(\omega M_{12}\right)\left(\omega M_{23}\right)\left(\omega M_{34}\right)}{Z_{11} Z_{22} Z_{33} Z_{44}+\left(\omega M_{12}\right)^{2} Z_{33} Z_{44}+\left(\omega M_{23}\right)^{2} Z_{11} Z_{44}+\left(\omega M_{34}\right)^{2} Z_{11} Z_{22}+\left(\omega M_{12}\right)^{2}\left(\omega M_{34}\right)^{2}} V_{\mathrm{S}}
\end{aligned}
$$

By applying Equations (2) to Equations (3) and (4), we obtain more compact expressions for these currents: 


$$
\begin{gathered}
I_{1}=\frac{1}{\Delta}\left(1+k_{23}^{2} Q_{2} Q_{3}+k_{34}^{2} Q_{3} Q_{4}\right) \frac{V_{\mathrm{S}}}{R_{1}+R_{\mathrm{S}}} \\
I_{4}=\frac{j}{\Delta}\left(k_{12} k_{23} k_{34} \sqrt{Q_{1} Q_{2}} \sqrt{Q_{2} Q_{3}} \sqrt{Q_{3} Q_{4}}\right) \frac{V_{\mathrm{S}}}{\sqrt{\left(R_{1}+R_{\mathrm{S}}\right)\left(R_{4}+R_{\mathrm{L}}\right)}}
\end{gathered}
$$

where:

$$
\Delta=\left[\left(1+k_{12}^{2} Q_{1} Q_{2}\right)\left(1+k_{34}^{2} Q_{3} Q_{4}\right)+k_{23}^{2} Q_{2} Q_{3}\right]
$$

To achieve impedance matching at the input port, we examine the input impedance $Z_{\mathrm{IN}}$ by looking into the source loop. Using:

$$
Z_{\mathrm{IN}}=V_{1} / I_{1}=\left(V_{\mathrm{S}}-R_{\mathrm{S}} I_{1}\right) / I_{1}
$$

we obtain:

$$
Z_{\mathrm{IN}}=\left(R_{1}+R_{\mathrm{S}}\right) \frac{1+k_{12}^{2} Q_{1} Q_{2}+k_{23}^{2} Q_{2} Q_{3}+k_{34}^{2} Q_{3} Q_{4}+k_{12}^{2} k_{34}^{2} Q_{1} Q_{2} Q_{3} Q_{4}}{1+k_{23}^{2} Q_{2} Q_{3}+k_{34}^{2} Q_{3} Q_{4}}-R_{\mathrm{S}}
$$

In the case of $R_{1}<<R_{\mathrm{S}}$, Equation (7) is simplified to:

$$
Z_{I N} \cong R_{\mathrm{S}}\left[\frac{k_{12}^{2} Q_{1} Q_{2}\left(1+k_{34}^{2} Q_{3} Q_{4}\right)}{1+k_{23}^{2} Q_{2} Q_{3}+k_{34}^{2} Q_{3} Q_{4}}\right]
$$

When one of the geometric parameters $(d, \rho, \theta)$ changes, it affects the coupling coefficients, thereby resulting in change of $Z_{\mathrm{IN}}$. A smart matching network senses this change in $Z_{\mathrm{IN}}$ so that the converted impedance $Z_{\mathrm{IN}}$,match is matched again to $R_{\mathrm{s}}$. Implementation of the matching network is described in Section 3.

At close distance, such as in an over-coupled region [8], we must consider the effect of cross-coupling on $Z_{\mathrm{IN}}$. The cross-coupling causes shifts in the frequencies of the transmission peaks. The frequency shift can be explained using the phase of the two modes [8], where the strength of the cross-coupling term determines the amount of the frequency shift. With the cross-coupling coefficients included, we solve Equation (1) for $I_{1}$ and obtain:

$$
Z_{\text {IN }}=\frac{\Delta Z}{Z_{23}\left(Z_{24} Z_{34}-Z_{23} Z_{44}\right)+Z_{33}\left(Z_{22} Z_{44}-Z_{24}^{2}\right)+Z_{34}\left(Z_{23} Z_{24}-Z_{22} Z_{34}\right)}-R_{\mathrm{S}}
$$

where:

$$
\begin{aligned}
& \Delta Z=Z_{12}^{2}\left(Z_{34}^{2}-Z_{33} Z_{44}\right)+Z_{23}^{2}\left(Z_{14}^{2}-Z_{11} Z_{44}\right)-Z_{34}^{2} Z_{11} Z_{22}-Z_{14}^{2} Z_{22} Z_{33} \\
& +Z_{13}^{2}\left(Z_{24}^{2}-Z_{22} Z_{44}\right)-Z_{24}^{2} Z_{11} Z_{33}+Z_{44}\left(Z_{11} Z_{22} Z_{33}+2 Z_{12} Z_{23} Z_{13}\right) \\
& +2 Z_{12} Z_{14}\left(Z_{24} Z_{33}-Z_{23} Z_{34}\right)+2 Z_{13} Z_{34}\left(Z_{14} Z_{22}-Z_{12} Z_{24}\right) \\
& +2 Z_{23} Z_{24}\left(Z_{34} Z_{11}-Z_{13} Z_{14}\right)
\end{aligned}
$$

Using Equation (9), it can be shown that the system model with cross-coupling creates imaginary parts in $Z_{\mathrm{IN}}$. 


\subsection{Parameter Extraction}

In our WPT system, the source and load loop coils each have a radius of $r_{1}=20 \mathrm{~cm}$. All coils are fabricated with copper of wire radius $a_{1}=0.15 \mathrm{~cm}$. Inductance $L_{\text {loop }}$ for the source and load loops is obtained using [21]:

$$
L_{\text {loop }}=\mu_{0} r_{1}\left[\ln \left(\frac{8 r_{1}}{a_{1}}\right)-1.75\right]
$$

where $\mu_{0}$ is the permeability of free space. Figure 2 shows the coil geometry of resonators 2 and 3. Inductance $L_{\text {spiral }}$ (in $\mu \mathrm{H}$ ) of this multi-turn flat spiral coil is calculated using the Wheeler formula [22]:

$$
L_{\text {spiral }}=\frac{r_{m}^{2} N^{2}}{2.54 \times\left(8 r_{m}+11 b\right)}
$$

where $b=5 \mathrm{~cm}$ is the depth of the winding, $r_{m}=27.5 \mathrm{~cm}$ is the mean radius, and $N=6$ is the number of turns, respectively.

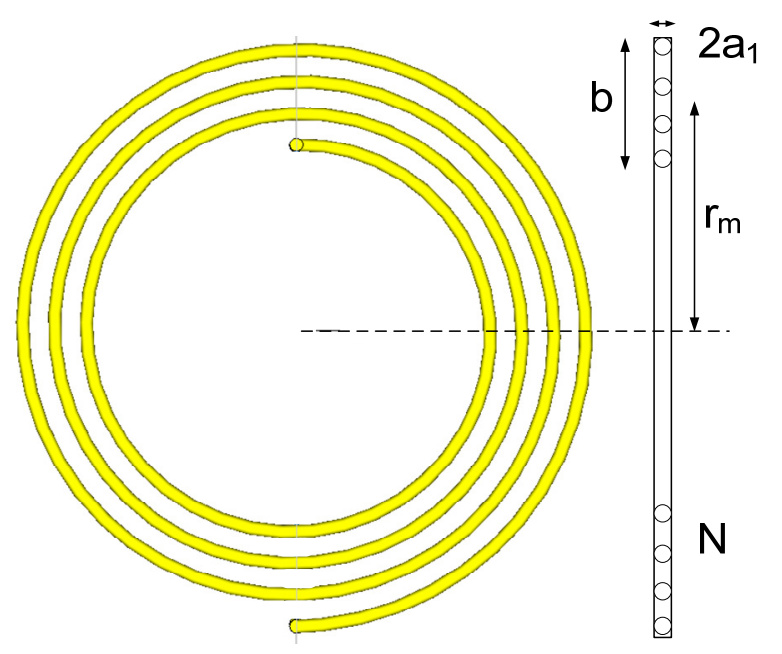

Figure 2. Dimension of multi-turn flat spiral for inductance calculation.

When the axes of the circular filaments are parallel, the mutual inductance $M$ is obtained using Maxwell's method [23]. For two parallel single-turn coils having radii $r_{m}$ and $r_{n}, M$ can be expressed as:

$$
\begin{gathered}
M\left(r_{m}, r_{n}, \rho, d\right)=\pi \mu_{0} \sqrt{r_{m} r_{n}} \int_{0}^{\infty} J_{1}\left(\sqrt{r_{m} / r_{n}} x\right) J_{1}\left(\sqrt{r_{n} / r_{m}} x\right) \\
\times J_{0}\left(\rho / \sqrt{r_{m} r_{n}} x\right) \exp \left(-d / \sqrt{r_{m} r_{n}} x\right) d x
\end{gathered}
$$

where $J_{0}$ and $J_{1}$ are Bessel functions of the zero-th and first order, respectively. This expression does not contain wire radius $a$; it is assumed that ratios $a / r_{m}$ and $a / r_{n}$ are sufficiently small [24]. Using Equation (12), we calculate the coupling coefficient $k_{i j}$,calc between the two multi-turn coils using:

$$
k_{i j, \text { calc }}(\rho, d)=\left[\sum_{m=1}^{N_{2}} \sum_{n=1}^{N_{3}} M\left(r_{m}, r_{n}, \rho, d\right)\right] / \sqrt{L_{i} L_{j}}
$$

where $N_{2}$ and $N_{3}$ are numbers of turns, and $L_{i}$ and $L_{j}$ are the inductances obtained using Equations (10) and (11). 
For lateral and angular misalignment between circular filaments, Grover [25,26]developed a method to calculate $M$ based on Neumann's formula. Using the method of vector potential, Babic et al. [27] developed a general mutual inductance formula requiring simple numerical integration. To reduce computational effort, we use this method to obtain the coupling coefficients.

To extract coupling coefficient $k_{i j}$,meas by experiment, we use the frequency splitting occurring in the near distance expressed as:

$$
k_{i j \text {, meas }}=\frac{f_{\mathrm{H}}^{2}-f_{\mathrm{L}}^{2}}{f_{\mathrm{H}}^{2}+f_{\mathrm{L}}^{2}}
$$

where $f_{\mathrm{H}}$ and $f_{\mathrm{L}}$ are the higher (odd mode) and lower (even mode) split frequencies of the magnetically-coupled resonators, respectively [28]. At great distance, the coupling is weak and the frequency splitting disappears. Because the WPT system of Figure 1 is a two-port network, we can capture the system characteristic using transmission coefficient $\left|\mathrm{S}_{21}\right|$. Then, $k_{i j}$,meas is extracted by fitting the system model of Figure $1 b$ to the measured $\left|S_{21}\right|$. Figure 3a shows measured frequency splitting versus distance. The splitting frequency ranges from $f_{\mathrm{L}}=5.92 \mathrm{MHz}$ to $\mathrm{f}_{\mathrm{H}}=8.13 \mathrm{MHz}$. Figure $3 \mathrm{~b}$ shows $\left|\mathrm{S}_{21}\right|$ versus distance $d$ measured using $f_{\mathrm{L}}, f_{\mathrm{H}}$, and $f_{0}=6.78 \mathrm{MHz}$, respectively. When $d$ is smaller than $40 \mathrm{~cm}$ (the boundary between the over-coupled and under-coupled regions [8]), frequency splitting occurs, and $\left|\mathrm{S}_{21}\right|$ is larger when measured at $f_{\mathrm{L}}$ than at either $f_{0}$ or $f_{\mathrm{H}}$.

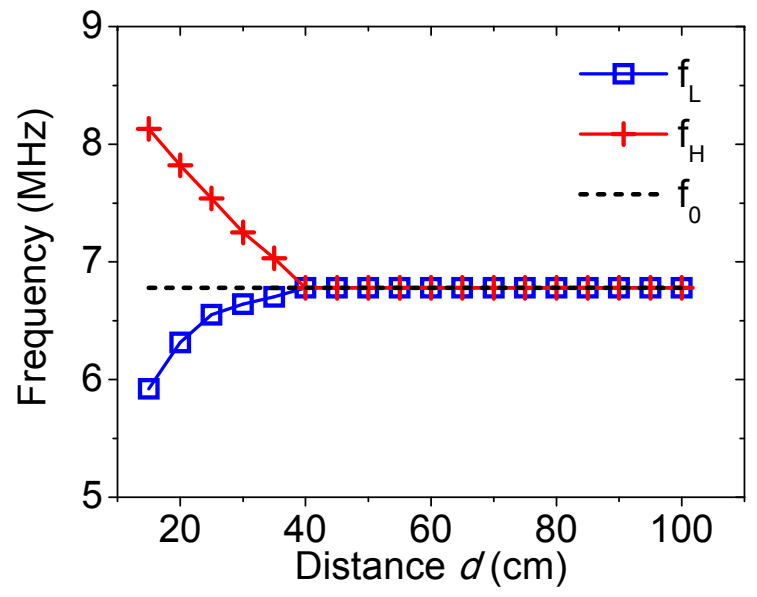

(a)

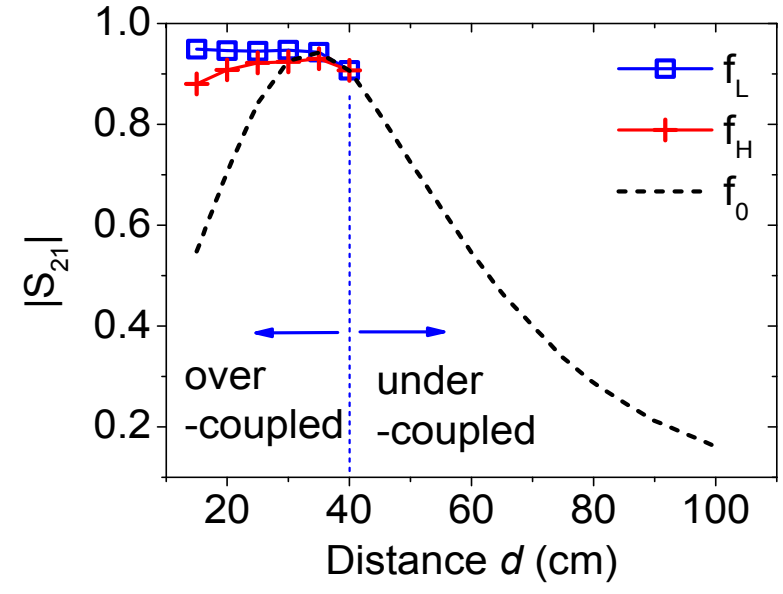

(b)

Figure 3. (a) Measured frequency splitting versus distance; and (b) measured $\left|\mathrm{S}_{21}\right|$ versus distance under the frequency-splitting phenomenon.

Figure 4a presents a comparison between the measured and calculated coupling coefficients $\left(k_{23}, k_{14}\right.$, and $k_{13}=k_{24}$ ) as functions of $d$ (without misalignment, $\rho=0 \mathrm{~cm}$ and $\theta=0^{\circ}$ ). The coupling coefficients decrease rapidly with $d$, and the calculated results show good agreement with the measured data. Figure $4 \mathrm{~b}$ shows the measured and calculated coupling coefficients as functions of $\rho$ for $d=30 \mathrm{~cm}$. Figure $4 \mathrm{c}$ shows the coupling coefficients as a function of $\theta$ for $d=40 \mathrm{~cm}$. The coupling coefficients decrease slowly for $\theta<60^{\circ}$, whereas they converge rapidly to 0 when $\theta$ approaches $90^{\circ}$ (for example, $k_{23}, k_{13}$, and $k_{14}$ are $2 \times 10^{-5}, 9 \times 10^{-6}$, and $7 \times 10^{-6}$, respectively, at $\theta=89.99^{\circ}$ ). 


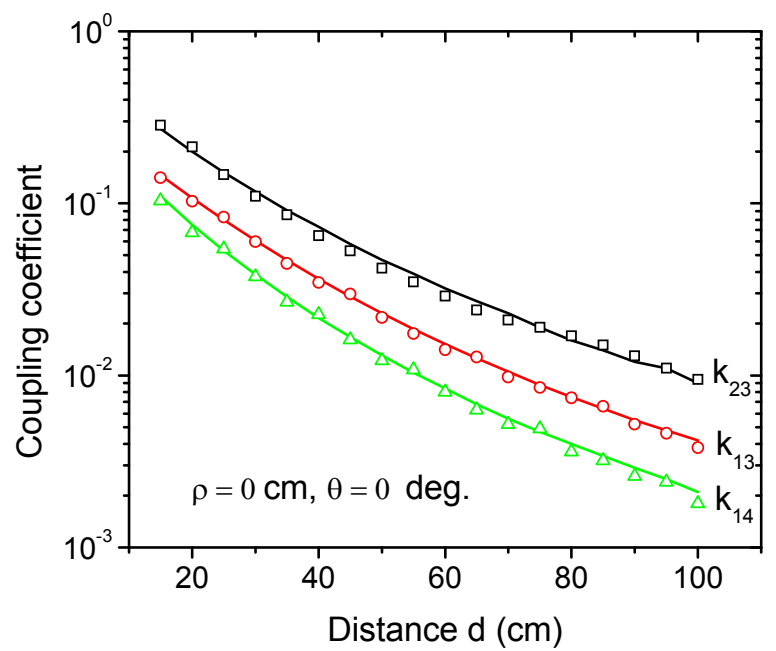

(a)

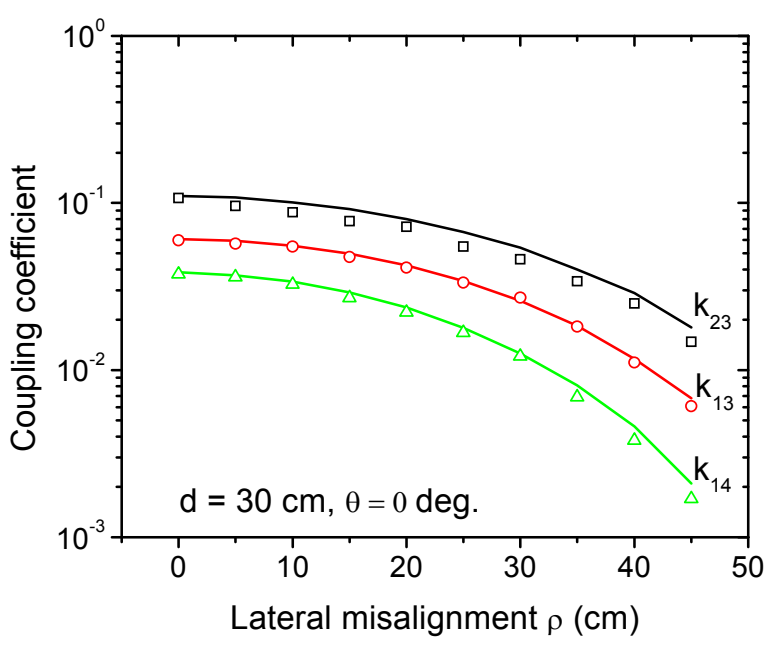

(b)

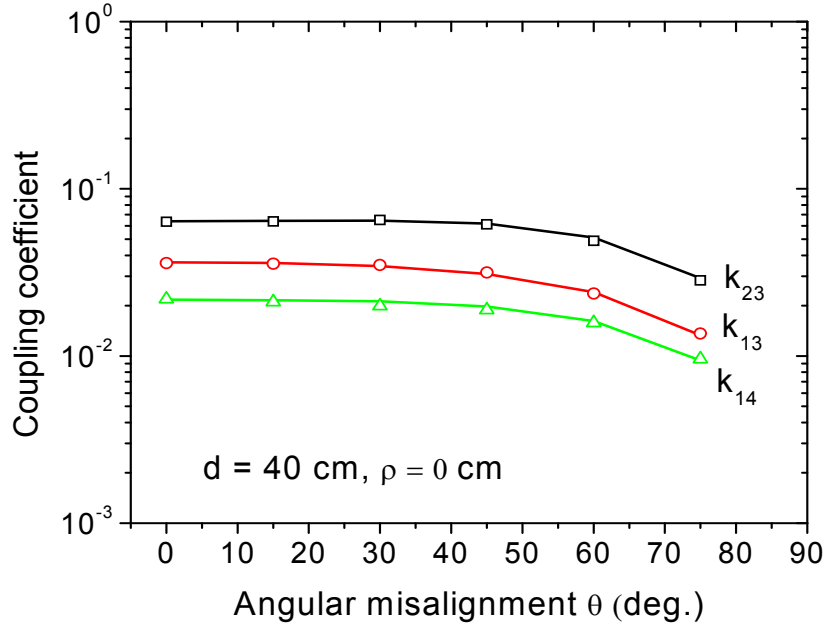

(c)

Figure 4. Measured (symbol) and calculated (line) coupling coefficients as functions of (a) distance; (b) lateral misalignment; and (c) angular misalignment.

Table 1 shows the measured electrical parameters of the coils using an Agilent 8714ES network analyzer (Santa Rosa, CA, USA). The data is measured at $f_{0}=6.78 \mathrm{MHz}$, which is the resonant frequency of the coils. For resonant frequency tuning, the values of the external variable capacitors in the source and load loops are carefully tuned using the network analyzer. For resonators 2 and 3, we use the self-capacitance existing in the resonator geometry [8]. In the experiment, we reduce resonator length by cutting it little by little until we obtain the resonant frequency. The calculated inductances using Equations (10) and (11) are $L_{\text {loop }}=1.31 \mu \mathrm{H}$ and $L_{\text {spiral }}=38.98 \mu \mathrm{H}$, which agree with the measured data within a $3.1 \%$ difference.

Table 1. Measured electrical parameters of coils.

\begin{tabular}{ccccc}
\hline Parameters & Inductance $(\boldsymbol{\mu H})$ & Resistance $(\boldsymbol{\Omega})$ & Resonant frequency $(\mathbf{M H z})$ & $\boldsymbol{Q}$ factor $@ \boldsymbol{f}_{\mathbf{0}}$ \\
\hline Source loop & 1.33 & 0.5 & 6.75 & 113 \\
Resonator 2 & 40.1 & 5.5 & 6.76 & 311 \\
Resonator 3 & 39.5 & 5.2 & 6.78 & 324 \\
Load loop & 1.35 & 0.4 & 6.73 & 144 \\
\hline
\end{tabular}




\section{Implementation}

\subsection{Tunable Matching Network}

Figure 5 shows the schematic of the tunable matching network. The matching network consists of a variable inductor $L_{\text {tune, switches }} \mathrm{SW}_{\mathrm{S}, k}$ and $\mathrm{SW}_{\mathrm{P}, k}$, and capacitor banks $C_{\mathrm{S}, k}$ and $C_{\mathrm{P}, k}(k=1-8)$. To realize a fine search step, $C_{\mathrm{S}, k}$ and $C_{\mathrm{P}, k}$ each consists of a set of eight capacitors $(10 \mathrm{pF}, 20 \mathrm{pF}$, $40 \mathrm{pF}, 50 \mathrm{pF}, 100 \mathrm{pF}, 200 \mathrm{pF}, 400 \mathrm{pF}$ and $500 \mathrm{pF}$ ) with $100 \mathrm{~V}$ ratings. By selecting a combination of switches $\mathrm{SW}_{\mathrm{S}, k}$ and $\mathrm{SW}_{\mathrm{P}, k}, C_{\mathrm{var} 1}$ and $C_{\mathrm{var} 2}$ change from $10 \mathrm{pF}$ to $1320 \mathrm{pF}$ in $10 \mathrm{pF}$ steps. Previous works used the capacitor matrix only [16-18]. To realize the flexible matching network, we include a variable inductor as well as the capacitor matrix.

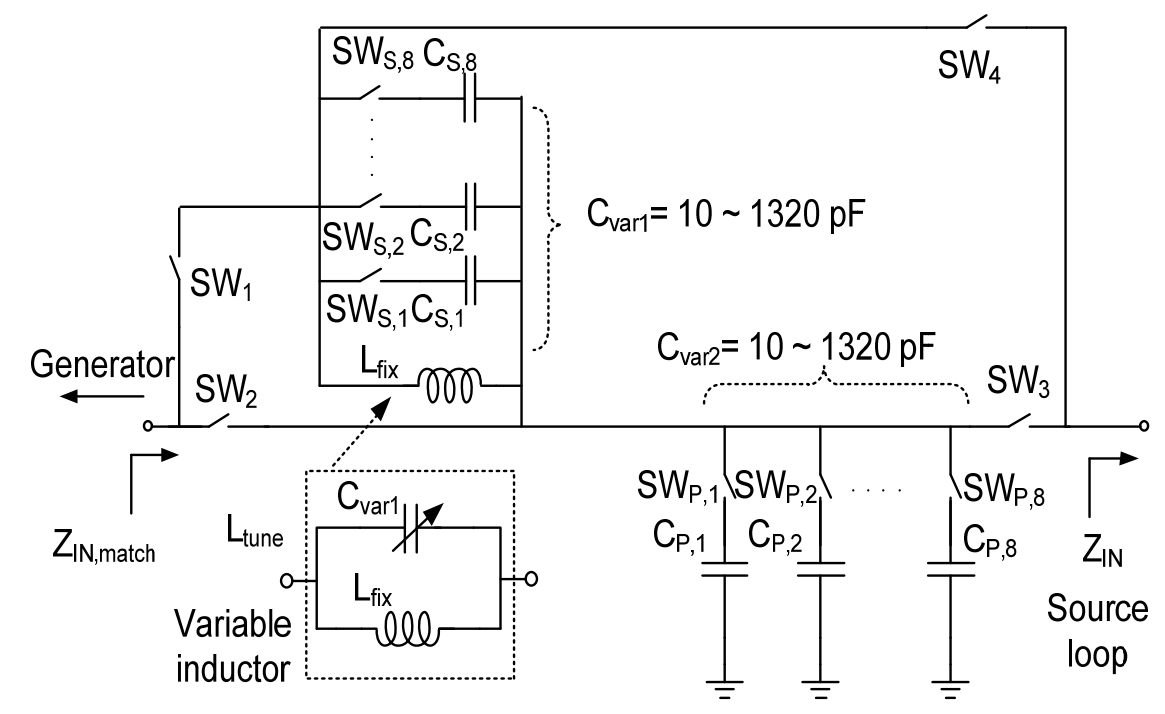

Figure 5. Schematic of tunable matching network; and circuit schematic of tunable inductor $L_{\text {tune }}$ is shown in the inset.

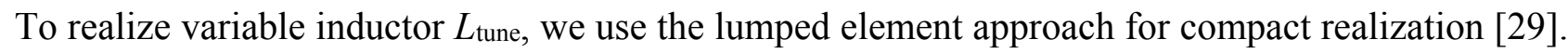
$L_{\text {tune }}$ consists of fixed inductor $L_{\text {fix }}$ and variable capacitor $C_{\text {var1 }}$ in a parallel configuration, as shown in the inset of Figure 5. From the complex impedance created by $L_{\text {fix }}$ and $C_{\text {var1 }}, L_{\text {tune }}$ can be expressed as:

$$
L_{\text {tune }}=\frac{L_{\mathrm{fix}}+L_{\mathrm{par}}}{1-\left(\omega / \omega_{\mathrm{c}}\right)^{2}}
$$

where:

$$
\omega_{\mathrm{c}}=1 / \sqrt{\left(C_{\mathrm{varl}}+C_{\mathrm{par}}\right)\left(L_{\mathrm{fix}}+L_{\mathrm{par}}\right)}
$$

is the resonance frequency of the variable inductor, $L_{\mathrm{par}}=60 \mathrm{nH}$ is the parasitic inductance from the board trace, and $C_{\mathrm{par}}=15 \mathrm{pF}$ is the parasitic capacitance (average value) of the relay switch. When $Z_{\mathrm{IN}}$ changes due to coil movement, the tunable matching network searches for values of $L_{\text {tune }}$ and $C_{\text {var2 }}$ for

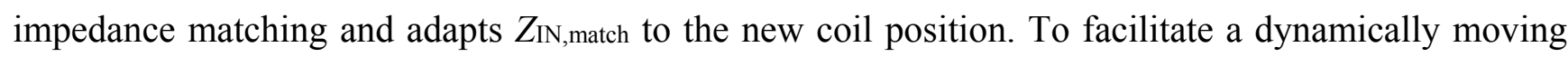
receiver, fast adaptation capability is desirable for the matching network. 
The switches are realized using an electrically controlled relay [30]. When the relay is actuated by $5 \mathrm{~V}$, the switching time is approximately $10 \mathrm{~ms}$. The relay can switch to as high as $250 \mathrm{~V}$ and $2 \mathrm{~A}$. We employ a

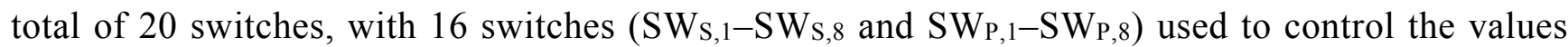
of $C_{\mathrm{var} 1}$ and $C_{\mathrm{var} 2}$. The four switches $\left(\mathrm{SW}_{1}-\mathrm{SW}_{4}\right)$ provide flexibility in realizing the matching network. With $\mathrm{SW}_{2}=\mathrm{SW}_{3}=\mathrm{ON}$ while other switches are OFF, the matching network is bypassed. The bypass configuration is used in the system calibration step (Figure 6).

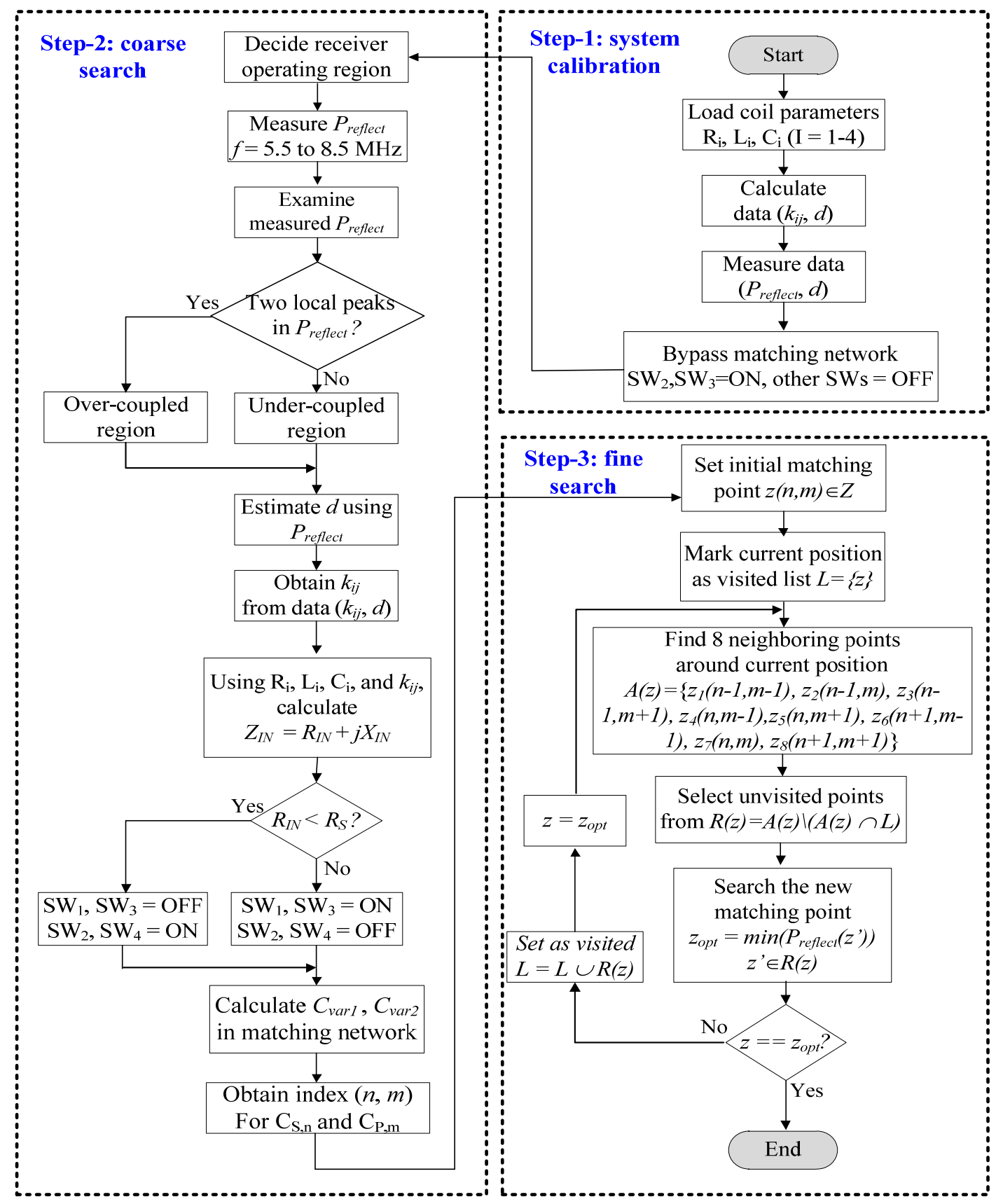

Figure 6. Flow chart showing the impedance searching method.

The port impedance of the components connected to the matching network corresponds to $R_{\mathrm{S}}=50 \Omega$. Although the current setup uses $50 \Omega$ due to constraints from the components and measurement equipment, the value of $R_{S}$ does not need to be $50 \Omega$. To allow for impedance matching in a general case of source impedance $Z_{\mathrm{S}}=R_{\mathrm{S}}+j \cdot X_{\mathrm{S}}$, the matching network provides two different configurations. 
With $\mathrm{SW}_{1}=\mathrm{SW}_{3}=\mathrm{ON}$ and $\mathrm{SW}_{2}=\mathrm{SW}_{4}=\mathrm{OFF}$, the series-L/shunt-C configuration is realized. Then, the desired values for $L_{\text {tune }}^{\prime}$ and $C_{\mathrm{var} 2}^{\prime}$ for impedance matching can be expressed as:

$$
\begin{gathered}
L_{\text {tune }}^{\prime}=\frac{1}{\omega}\left(\sqrt{\left.\frac{R_{\mathrm{S}}}{\operatorname{Re}\left[Z_{\mathrm{IN}}\right]} \Delta R_{1}-X_{\mathrm{S}}\right)}\right. \\
C_{\mathrm{var} 2}^{\prime}=\frac{1}{\omega} \frac{\operatorname{Re}\left[Z_{\mathrm{IN}}\right]-R_{\mathrm{S}}}{\sqrt{R_{\mathrm{S}} \operatorname{Re}\left[Z_{\mathrm{IN}}\right]} \Delta R_{1}-R_{\mathrm{S}} \operatorname{Im}\left[Z_{\mathrm{IN}}\right]}
\end{gathered}
$$

where:

$$
\Delta R_{1}=\sqrt{\operatorname{Re}^{2}\left[Z_{\text {IN }}\right]+\operatorname{Im}^{2}\left[Z_{\mathrm{IN}}\right]-R_{\mathrm{S}} \operatorname{Re}\left[Z_{\mathrm{IN}}\right]}
$$

With $\mathrm{SW}_{2}=\mathrm{SW}_{4}=\mathrm{ON}$ and $\mathrm{SW}_{1}=\mathrm{SW}_{3}=\mathrm{OFF}$, the shunt-C/series-L configuration is realized, and the desired values for $L_{\text {tune }}^{\prime}$ and $C_{\mathrm{var} 2}^{\prime}$ for impedance matching can be expressed as:

$$
\begin{gathered}
L_{\text {tune }}^{\prime}=\frac{1}{\omega}\left\{\frac{X_{\mathrm{S}}\left(R_{\mathrm{S}}^{2}+X_{\mathrm{S}}^{2}\right)-\left(X_{\mathrm{S}}+\Delta R_{2}\right) R_{\mathrm{S}} \operatorname{Re}\left[Z_{\mathrm{IN}}\right]}{\left(R_{\mathrm{S}}^{2}+X_{\mathrm{S}}^{2}\right)-\left(X_{\mathrm{S}}+\Delta R_{2}\right) X_{\mathrm{S}}}-\operatorname{Im}\left[Z_{\mathrm{IN}}\right]\right\} \\
C_{\mathrm{var} 2}^{\prime}=\frac{1}{\omega} \frac{\Delta R_{2}+X_{\mathrm{S}}}{R_{\mathrm{S}}^{2}+X_{\mathrm{S}}^{2}}
\end{gathered}
$$

where:

$$
\Delta R_{2}=\sqrt{R_{\mathrm{S}}\left(R_{\mathrm{S}}^{2}+X_{\mathrm{S}}^{2}-R_{\mathrm{S}} \operatorname{Re}\left[Z_{\mathrm{IN}}\right]\right) / \operatorname{Re}\left[Z_{\mathrm{IN}}\right]}
$$

By substituting Equations (16) and (18) into Equation (15), we calculate the initial values for $C_{\text {varl: }}$ :

$$
C_{\mathrm{var} 1}=\frac{1}{\omega^{2}}\left(\frac{1}{L_{\mathrm{fix}}+L_{\mathrm{par}}}-\frac{1}{L_{\mathrm{tune}}^{\prime}}\right)-C_{\mathrm{par}}
$$

The fine-search step (Figure 6) refines the values of $C_{\mathrm{var}}$ and $C_{\mathrm{var}}$ for impedance matching; i.e., $L_{\text {tune }} \rightarrow L_{\text {tune }}^{\prime}$ and $C_{\mathrm{var} 2} \rightarrow C_{\mathrm{var} 2}^{\prime}$.

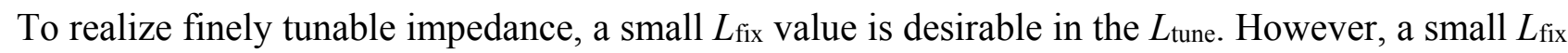
leads to narrow inductance and impedance tuning ranges. To determine a suitable value for $L_{\text {fix }}$, we examine $Z_{\mathrm{IN}}$ and the magnitude of the reflection coefficient $\Gamma_{\mathrm{IN}}=\left(Z_{\mathrm{IN}}-50\right) /\left(Z_{\mathrm{IN}}+50\right)$ of the WPT system. Figure 7 shows the measured $Z_{\mathrm{IN}}$ when $d$ is varied from $15 \mathrm{~cm}$ to $100 \mathrm{~cm}$ in $5 \mathrm{~cm}$ steps. $Z_{\mathrm{IN}}$ is measured by connecting the source loop to port 1 of a network analyzer. $Z_{\mathrm{IN}}$ is capacitive in the near distance, whereas it changes to inductive when $d>40 \mathrm{~cm}$ in our system. When $d$ changes from $15 \mathrm{~cm}$ to $100 \mathrm{~cm},\left|\Gamma_{\mathrm{IN}}\right|$ varies from 0.242 to 0.908 . Additionally, shown in Figure 7 , are the measured $Z_{\mathrm{IN}}$ values when misalignment $\rho$ changes from $5 \mathrm{~cm}$ to $45 \mathrm{~cm}$ in $5 \mathrm{~cm}$ steps at $d=15 \mathrm{~cm}$. In this case, $\left|\Gamma_{I N}\right|$ varies from 0.066 to 0.924 . Considering the trade-off between the achievable impedance range and impedance step, we choose $L_{\mathrm{fix}}=440 \mathrm{nH}$. Using the $L_{\text {fix }}$ value, the tunable matching network covers the $\left|\Gamma_{\mathrm{IN}}\right|$ range of our WPT system. For the $Z_{\mathrm{IN}}$ simulation, we input both the coil parameters (Table 1) and coupling coefficients (Figure 4) into the system model of Figure 1b. We observe good agreement between the measured and simulated $Z_{\mathrm{IN}}$, indicating the accuracy of the extracted parameters. 


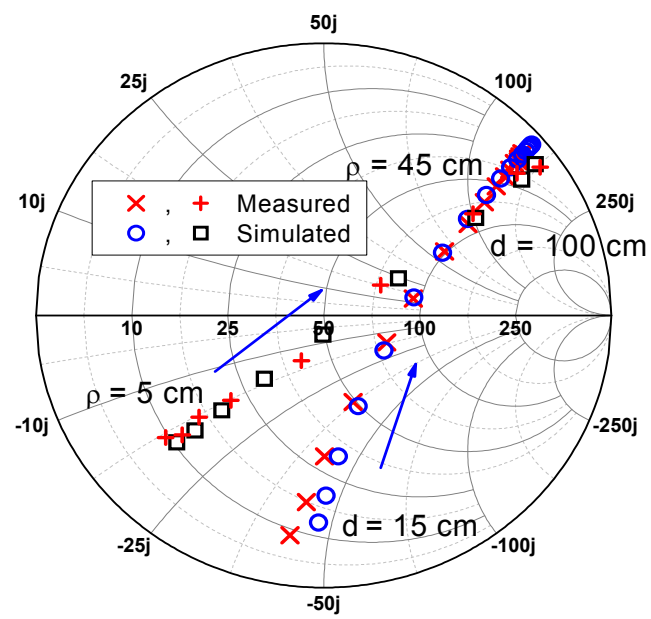

Figure 7. Measured and simulated $Z_{\mathrm{IN}}$ for distance and alignment changes.

\subsection{Three-Step Searching Method}

To select a search method for the values of $C_{\mathrm{var}}$ and $C_{\mathrm{var}}$, we examine the two-port network of the WPT system (Figure 1) using $\left|S_{21}\right|$. Figure 8 shows the simulated $\left|S_{21}\right|$ values for $d=15 \mathrm{~cm}, 40 \mathrm{~cm}$, and $80 \mathrm{~cm}$. The results show a single peak with a smooth slope, which agrees with a previous report [18]. Examining all impedance points, however, is too time consuming to use in an environment in which coils move dynamically; in our system, the time required to actuate a relay switch is approximately $10 \mathrm{~ms}$. Because $C_{\text {var1 }}$ and $C_{\text {var2 }}$ change from $10 \mathrm{pF}$ to $1320 \mathrm{pF}$ in $10 \mathrm{pF}$ steps, the dimensions of the search matrix for $C_{\text {var1 }}$ and $C_{\text {var2 }}$ will be $132 \times 132=17,424$. Then, the required time is approximately 3 min. To reduce search time and quickly adapt to dynamically moving coils, we propose an efficient searching method for the tunable matching network.

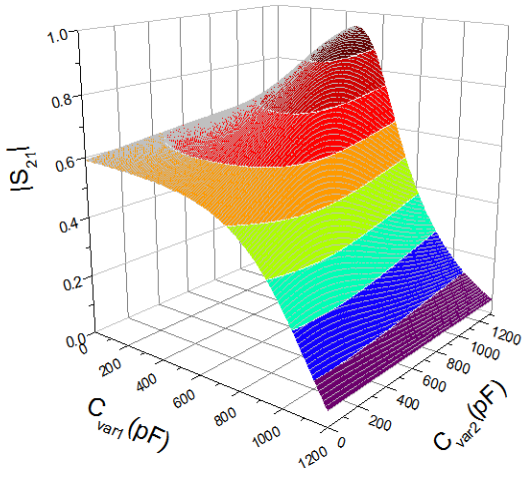

(a)

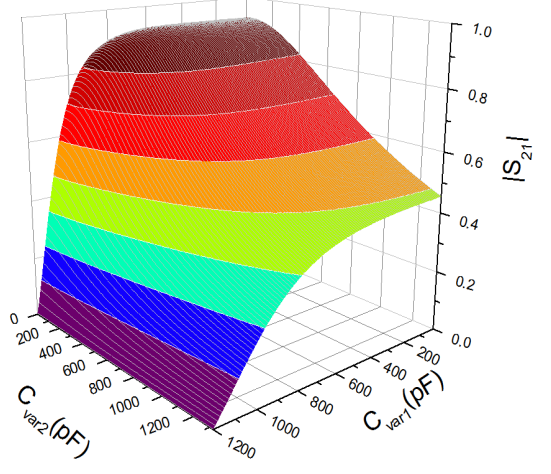

(b)

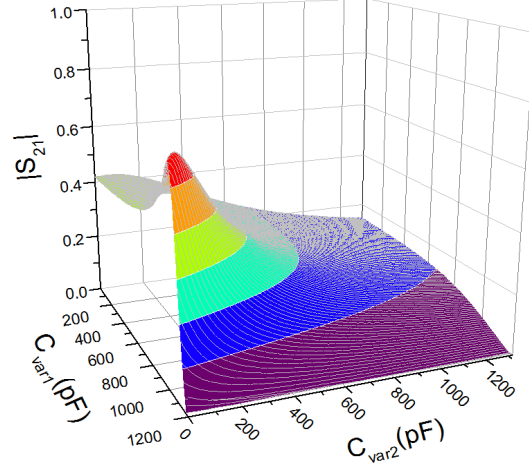

(c)

Figure 8. $\left|\mathrm{S}_{21}\right|$ depending on capacitor values: (a) $d=15 \mathrm{~cm}$; (b) $d=40 \mathrm{~cm}$; and (c) $d=80 \mathrm{~cm}$.

Figure 6 presents a flowchart of the proposed searching method. The method consists of three steps: system calibration, coarse search, and fine search. The operations performed in each step are as follows:

Step 1: The system calibration step is performed one time to calculate and measure the system parameters. Using the coil parameters in (13), $k_{i j, \text { calc }}$ as functions of $d$ are obtained. With the matching network in the bypass configuration $\left(\mathrm{SW}_{2}=\mathrm{SW}_{3}=\mathrm{ON}\right.$ while other switches are OFF), the reflected 
power $P_{\text {reflect }}$ at the input of the matching network is measured as $d$ is changed from $15 \mathrm{~cm}$ to $100 \mathrm{~cm}$ in $5 \mathrm{~cm}$ steps. The data are used to calculate the initial value of $Z_{\mathrm{IN}}$ for Step 2.

Step 2: In the coarse-search step, the distance $d$ is estimated to obtain an initial value for the impedance search. The receiver (resonator 3 and load loop) can be positioned in either an over- or under-coupled region. When we use a single frequency $f_{0}=6.78 \mathrm{MHz}$, frequency splitting occurs and two distances can exist for which $\left|\mathrm{S}_{21}\right|$ (thus, the reflected power $P_{\text {reflect }}$ ) is the same, as shown in Figure $3 b$. Therefore, the WPT system cannot distinguish between the two regions. To detect the region where the receiver is positioned, we measure $P$ reflect while scanning the frequency in the range from $5.5 \mathrm{MHz}$ to 8.5 MHz (a low power level may be used during frequency scanning). At a near distance $d$, belonging to the over-coupled region, frequency splitting creates multiple transmission peaks [8]. In the case in which there are more than two local minimum peaks in $P_{\text {reflect, }}$ therefore, the system determines that the receiver is positioned in an over-coupled region. With increasing $d$, coupling decreases until the transmission peaks converge. Therefore, at a distance $d$, belonging to the under-coupled region, $P_{\text {reflect }}$ has one minimum peak. By detecting the number of transmission peaks, the region (over- or under-coupled) is identified. Then, the distance to the receiver is estimated by comparing the measured $P_{\text {reflect }}$ with the data obtained in Step 1. Using the estimated distance, the corresponding coupling coefficients are obtained using Figure 4a. By combining them with the coil parameters shown in Table 1, we calculate $Z_{i j}(i, j=1-4)$ and $Z_{\mathrm{IN}}$ using Equations (2) and (9), respectively. Using Equations (16)-(19) and $Z_{\mathrm{IN}}$, we obtain the values of $L_{\text {tune }}^{\prime}$ and $C_{\text {var } 2}^{\prime}$ for the matching network.

Step 3: In the fine-search step, the accuracy of the impedance matching is improved by refining the values in the matching network. The method used in this step is based on the hill-climb searching technique [31], which has been modified to enhance searching speed. In this step, the routine continuously evaluates the neighboring points in the direction of minimizing $P_{\text {reflect }}$ until a valley point is found. To speed up the searching process, previously visited points are marked and eliminated during the evaluation step. A detailed description of the proposed method is as follows. Let the two-dimensional values of $C_{\mathrm{var} 1}$ and $C_{\mathrm{var} 2}$ (with 10 -pF steps) be mapped to row indices $n$ and column indices $m$ of square-matrix $Z(n, m=1-132)$. Let $z(n, m)$ be the current location at row $n$ and column $m$ in $Z(z \in Z)$. Let $L$ be the list of points that have been visited during the searching process in $Z$. Then, the system performs the process as follows:

(1) Using the matching component values calculated in Step 2, set the initial starting point $z(n, m) \in Z$.

(2) Add point $z$ to the list of visited points $L$, or $L=\{z\}$.

(3) Determine the set of eight neighboring points $A(z)$ based on current location $z$

(4) Find the set of unvisited neighboring points $R(z)$ using $A(z)$ and list $L$.

(5) Using the detected $P_{\text {reflect }}$ at the coupler, find the best matching point $z_{\text {opt }}$ among the eight neighboring points in $R(z)$.

(6) Check whether $z_{\text {opt }}$ remains unchanged. If yes, the system process ends. If no, add the already examined points $R(z)$ to the list $L$; i.e., $L=L \mathrm{U}\left\{\mathrm{z}^{\prime} \mid \mathrm{z}^{\prime} \in R(z)\right\}$.

(7) Assign new starting point $z$ using local optimal point $z_{\text {opt }}$ for the next loop, and proceed to Step 3 (3). 


\section{Experimental Results}

Figure 9 presents a system block diagram of the proposed WPT. The system includes a signal generator, a radio-frequency (RF) power amplifier [32], a $20 \mathrm{~dB}$ directional coupler [33], a power detector [34], a tunable matching network, switch drivers, and a control unit. The RF power amplifier has $50-\Omega$ output impedance $\left(R_{\mathrm{S}}=50 \Omega\right)$, providing an available power $P_{\text {avail }}=30 \mathrm{dBm}\left(20 V_{\mathrm{p}-\mathrm{p}}\right.$ in $50 \Omega$ load $)$. An Agilent N1914A power meter and 8481B power sensor $\left(R_{\mathrm{L}}=50 \Omega\right)$ are used to measure the output power $P_{\text {out. }}$ The coupler and power detector are used to measure reflected power $P_{\text {reflect. Through the }}$ coupled reflected port, $P_{\text {reflect }}$ is scaled down so that it is within the dynamic input range $(-50 \mathrm{dBm}$ to $20 \mathrm{dBm}$ ) of the detector. The detector generates a linear voltage from $2.3 \mathrm{~V}$ to $0.5 \mathrm{~V}$, which is inversely proportional to power.

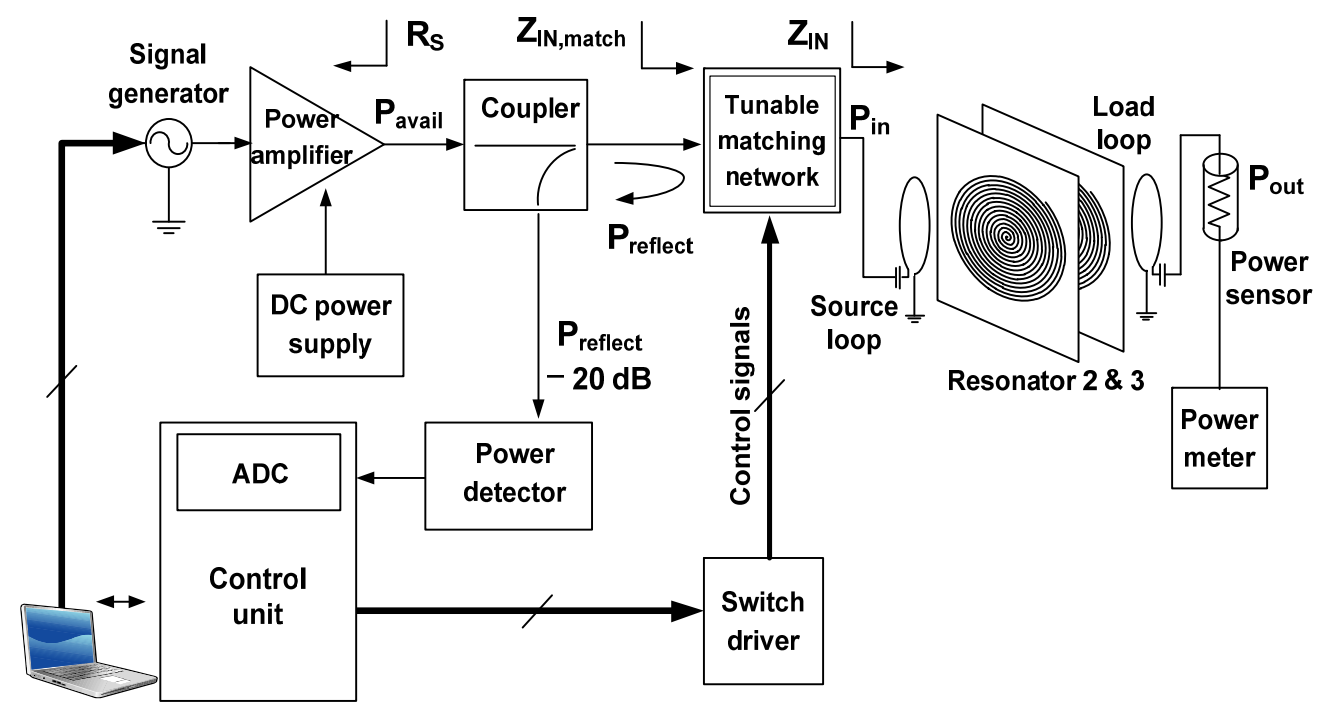

Figure 9. Block diagram of the proposed WPT system.

The control unit MSP430 includes a 10-bit analog-to-digital converter (ADC), which coverts this voltage to digitized data. Based on the data, the control unit executes the impedance searching routine

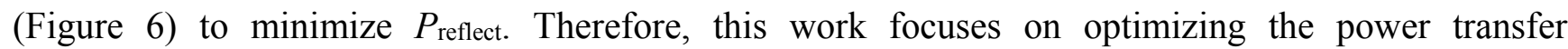
(transmission) efficiency without a rectifier at the load. The transmission efficiency is defined by the ratio of $P_{\text {out }}$ to $P_{\text {avail. }}$ For high-power applications, system energy efficiency, which includes the loss in the power source, is usually considered. Under the impedance matching condition, half of the power is dissipated in the power source, limiting the system efficiency to $50 \%$; reducing the loss in the power source is desirable [1]. In this work, however, design of a low-loss power source, i.e., an RF power amplifier, is out of scope. Furthermore, this work emphasizes enabling the geometric freedom envisioned for mobile devices. Therefore, we consider transmission efficiency for low-power devices operating in midrange applications, in which the loss of the power source is not of primary concern [1].

Figure 10 shows the experimental setup for the proposed WPT system. The coil structure is similar to one used in a previous work [35]; however, the resonant frequency is tuned to $6.78 \mathrm{MHz}$ in this work. The dynamic impedance-matching capability of the fabricated WPT system is characterized under the change of three geometric parameters: distance $d$, lateral misalignment $\rho$, and angular misalignment $\theta$. 


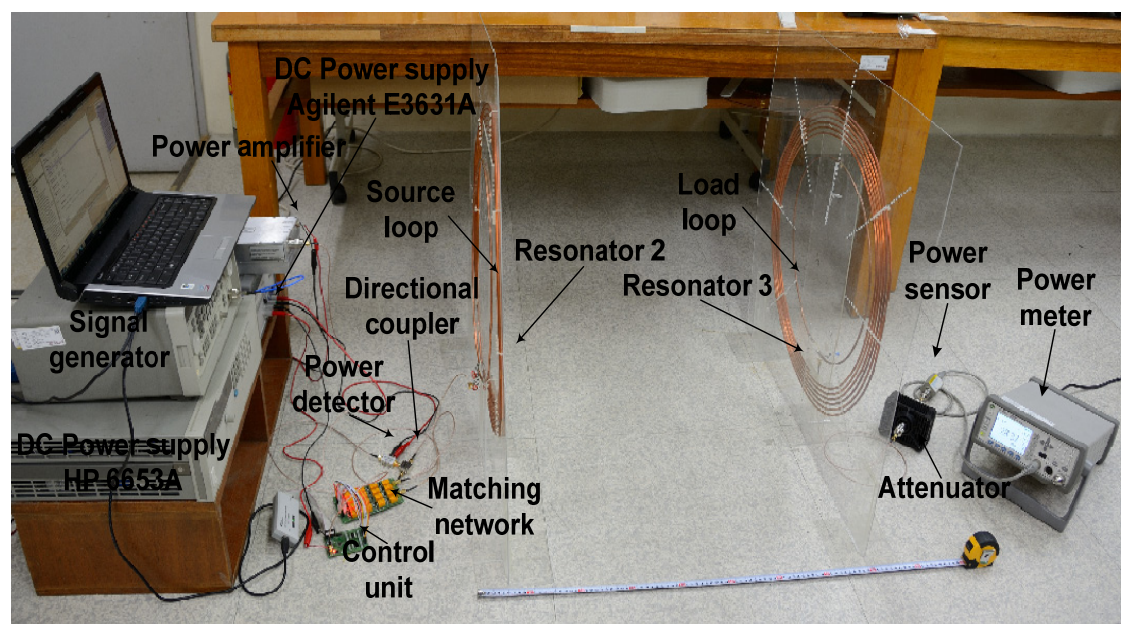

Figure 10. Experimental setup for the proposed WPT system.

\subsection{Distance Change}

Figure 11 shows measured impedance $Z_{\mathrm{IN} \text {,match }}$ at the input port of the matching network (Figure 9). When the system examines the eight neighboring points around the current location, the value of impedance $z_{\text {opt }}$ in the fine search (Step 3) is recorded. The impedance is measured at three distances ( $d=15 \mathrm{~cm}, 40 \mathrm{~cm}$, and $80 \mathrm{~cm}$ ) with $\rho=0 \mathrm{~cm}$ and $\theta=0^{\circ}$. If only the fine-search step is used, the impedance searching starts from the center of the search matrix (this is called a one-step method). A three-step method uses system calibration (Step 1), coarse search (Step 2), and fine search (Step 3).

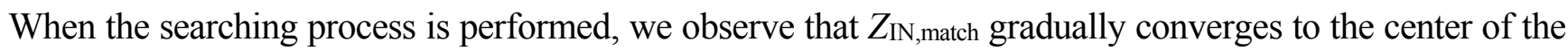

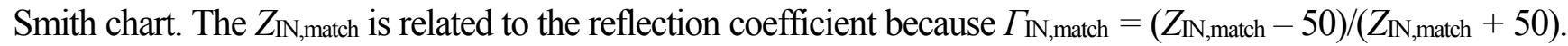
At $d=80 \mathrm{~cm},\left|\Gamma_{\mathrm{IN}, \text { match }}\right|$ improves from $-0.57 \mathrm{~dB}$ to $-25.6 \mathrm{~dB}$ using the one-step method. Using the three-step method, it improves from $-14.9 \mathrm{~dB}$ to $-25.6 \mathrm{~dB}$. The final $\left|\Gamma_{\mathrm{IN}, \text { match }}\right|$ is $-28.1 \mathrm{~dB}$ and $-44.8 \mathrm{~dB}$ at $d=15 \mathrm{~cm}$ and $40 \mathrm{~cm}$, respectively. These results indicate that the proposed tunable matching network achieves good impedance matching.

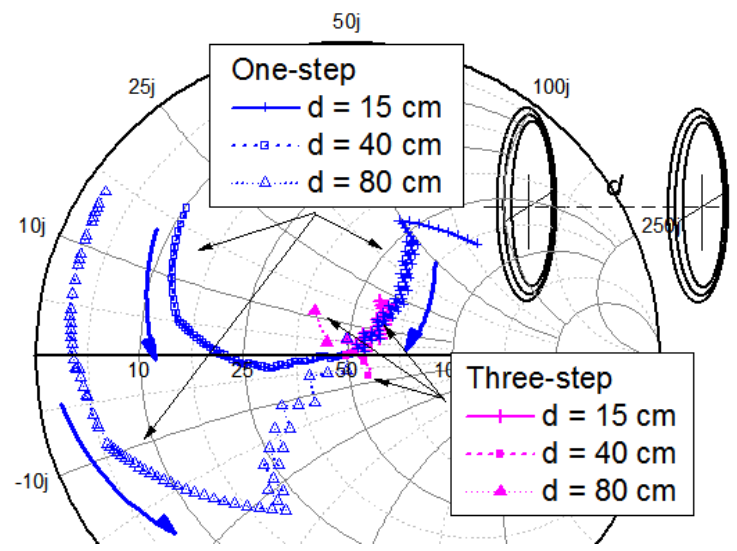

Figure 11. Measured trajectories of the input impedance for three distances.

When we compare the results of the two methods, we note that, compared to the one-step method, the initial starting point is closer to the center of the Smith chart when the three-step method is used. Thus, the trajectory of $Z_{\mathrm{IN} \text {,match }}$ is significantly shorter, indicating a faster searching capability. 
The number of examined points (including the eight neighboring points around $z_{\mathrm{opt}}$ ) during the fine search is equal to the number of relay switching. Using the one-step method, the number of points are 250,221 , and 307 at $d=15 \mathrm{~cm}, 40 \mathrm{~cm}$, and $80 \mathrm{~cm}$, respectively. Using the three-step method, it is 94 , 47,18 at $d=15 \mathrm{~cm}, 40 \mathrm{~cm}$, and $80 \mathrm{~cm}$, respectively. For practical application of the WPT system, operating lifetime should be considered. Based on the datasheet [30], the relay allows the maximum $10^{8}$ number of switching operations before end-of-lifetime. Based on this data, we can estimate the allowed number of impedance matching operations. Compared to the one-step method, the number of relay switching operation is significantly reduced using the three-step method, extending the operating lifetime of the WPT system.

To examine the searching speed, we measure the output of power detector $V_{\text {detect }}$ as a function of time. Figure 12 shows the measured results at three distances: $d=15 \mathrm{~cm}, 40 \mathrm{~cm}$, and $80 \mathrm{~cm}$. While the impedance matching is performed, $V_{\text {detect }}$ gradually increases with time $\left(\left|\Gamma_{\mathrm{IN}, \text { match }}\right|\right.$ decreases with time). Using the one-step method, the searching time is $2-3 \mathrm{~s}$. Using the three-step method, the times to reach the final point are $0.94 \mathrm{~s}, 0.47 \mathrm{~s}$, and $0.18 \mathrm{~s}$ for $d=15 \mathrm{~cm}, 40 \mathrm{~cm}$, and $80 \mathrm{~cm}$, respectively. In the case in which all impedance points are sequentially examined, the required time is approximately $180 \mathrm{~s}$. It is evident that the three-step method significantly reduces the searching time: using the proposed three-step method, the searching time is reduced more than 180 times.

The detector output $V_{\text {detect }}$ provides an alternative means for assessing the accuracy of impedance matching. Using the datasheet in [34], the measured $V_{\text {detect }}$ is converted to $P_{\text {reflect. From the relationship }}$ between $\Gamma_{\mathrm{IN} \text {,match }}$ and $P_{\text {reflect, }}$ we obtain:

$$
\left|\Gamma_{\text {IN,match }}\right|=\sqrt{\frac{P_{\text {reflect }}}{P_{\text {avail }}-I L_{\text {coupler }}}}
$$

where $I L_{\text {coupler }}=0.8 \mathrm{~dB}$ is the insertion loss of the coupler. When the three-step method finishes impedance matching, $\left|\Gamma_{\mathrm{IN}, \text { match }}\right|$ obtained using Equation $(21)$ is $-26.1 \mathrm{~dB},-42.7 \mathrm{~dB}$, and $-25.4 \mathrm{~dB}$ for $d=15 \mathrm{~cm}, 40 \mathrm{~cm}$, and $80 \mathrm{~cm}$, respectively. These results indicate good impedance matching, and correlate well with the results obtained using $Z_{\mathrm{IN} \text {,match. }}$

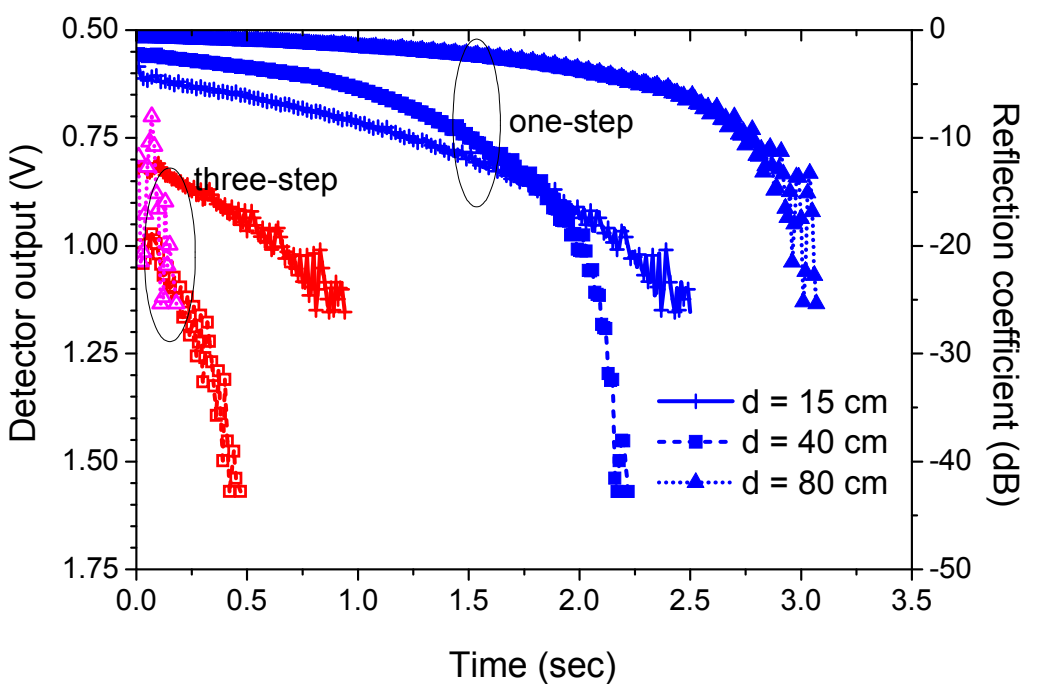

Figure 12. Measured detector output and reflection coefficient versus time at three distances: $d=15 \mathrm{~cm}, 40 \mathrm{~cm}$, and $80 \mathrm{~cm}$. 
Efficiency improvement with the proposed method is measured as a function of $d$. To examine the impedance matching effect, we measure the transmission efficiency. Considering that small mobile receivers typically do not have sufficient power and space for the system of a tunable matching network, the matching network is realized in the transmitter. Therefore, the impedance mismatch effect at the load loop of the receiver is removed by using the method in [35]. Figure 13 presents a comparison of the efficiency versus $d$. Without a matching network, the efficiency rapidly degrades when the distance deviates from the optimum coupling distance of approximately $35 \mathrm{~cm}$. However, using the proposed tuning method, efficiency is significantly improved; it increases by $57.5 \%$ and $47.1 \%$ at $d=15 \mathrm{~cm}$ and $d=70 \mathrm{~cm}$, respectively. In the near distance $(d<35 \mathrm{~cm})$, efficiency is higher than $88 \%$ with a maximum value of $89.7 \%$. These results additionally demonstrate the measured efficiency using the mechanical tuning method [35]. Using Equation (8), we obtain the condition for

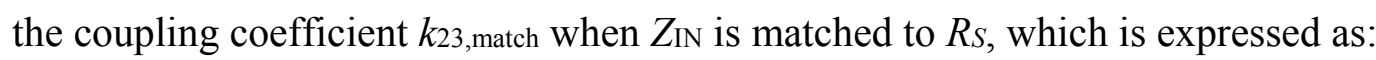

$$
k_{23, \text { match }} \cong \frac{1}{\sqrt{Q_{2} Q_{3}}} \sqrt{\left(k_{12}^{2} Q_{1} Q_{2}-1\right)\left(k_{34}^{2} Q_{3} Q_{4}+1\right)}
$$

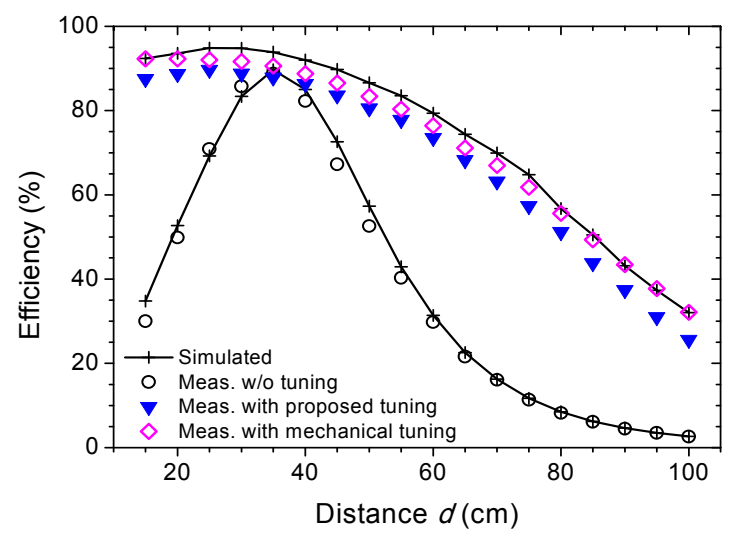

Figure 13. Efficiency comparison with and without a matching network.

For given coil parameters, the condition Equation (22) specifies the $k_{12}$ and $k_{34}$ required to achieve

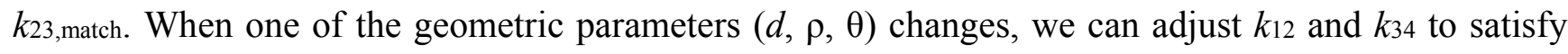
Equation (22). The insertion loss of the matching network is $0.45 \mathrm{~dB}$ (average value). Because of the insertion loss, the peak efficiency achieved using the proposed tuning is $2.3 \%$ lower than the one obtained using mechanical tuning. However, changing $k_{12}$ and $k_{34}$ by moving coils is not convenient; moreover, it is rather slow in practice. The measured results show that the efficiency achieved using the proposed tuning method closely follows that of the mechanical tuning method. The results demonstrate that the slow mechanical tuning can be replaced with the fast electric tuning capability of the proposed WPT.

\subsection{Lateral Misalignment}

Figure 14 shows the measured trajectory of $Z_{\mathrm{IN} \text {, match }}$ as misalignment ranges from $\rho=0 \mathrm{~cm}$ to $40 \mathrm{~cm}$ in $10 \mathrm{~cm}$ steps. In the proposed WPT system, this misalignment is not explicitly detected (only $d$ is estimated using $P_{\text {reflect }}$ in Step 2). Therefore, there will be error in the estimated $Z_{\mathrm{IN}}$. At $\rho=10 \mathrm{~cm}$, the measured $Z_{\mathrm{IN}}$ is $12.6-j 24.4 \Omega$, whereas the estimated value is $8.6-j 16.8 \Omega$. 


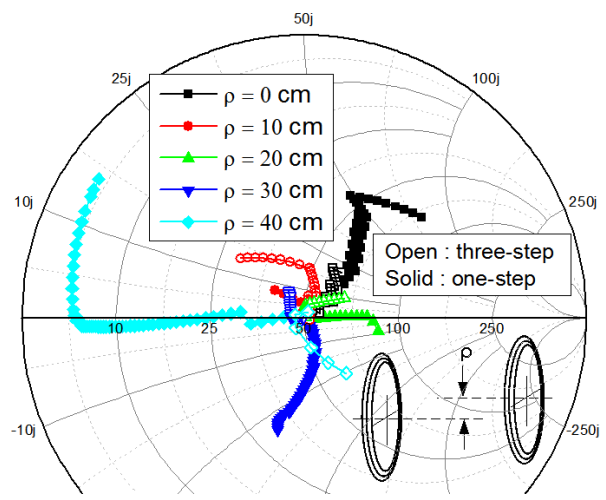

Figure 14. Trajectory of the measured input impedance for different misalignments from $\rho=0 \mathrm{~cm}$ to $40 \mathrm{~cm}$ for $d=15 \mathrm{~cm}$.

Although the estimated $Z_{\mathrm{IN}}$ differs somewhat from the actual value, the results, presented in Figure 14,

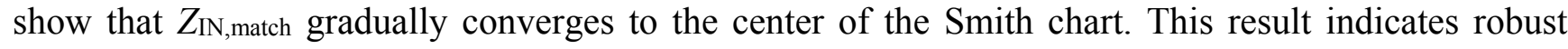
searching capability that is successful in dealing with mismatch caused by $\rho$. In addition, we note that the $Z_{\mathrm{IN} \text {,match }}$ trajectory of the three-step method is shorter than that of the one-step method. At $\rho=40 \mathrm{~cm}$, $\left|\Gamma_{\mathrm{IN}, \mathrm{match}}\right|$ improves from $-12.2 \mathrm{~dB}$ to $-31.9 \mathrm{~dB}$ (and from $-1.1 \mathrm{~dB}$ to $-32.0 \mathrm{~dB}$ ) using the three-step method (and using the one-step method). In the range where $\rho$ changes from $0 \mathrm{~cm}$ to $40 \mathrm{~cm}$, the worst case of measured $\left|\Gamma_{\mathrm{IN}, \text { match }}\right|$ at the final point is $-28.1 \mathrm{~dB}$ for $\rho=0 \mathrm{~cm}$, showing a good impedance match.

Figure 15 shows the measured $V_{\text {detect }}$ and $\left|\Gamma_{\mathrm{IN} \text {,match }}\right|$ as functions of time. As the impedance matching process is performed, $\left|\Gamma_{\mathrm{IN}, \text { match }}\right|$ gradually decreases with time. In the range where $\rho$ changes from $0 \mathrm{~cm}$ to $40 \mathrm{~cm}$, the worst case of measured $\left|\Gamma_{\mathrm{IN} \text {,match }}\right|$ using the three-step method is $-26.2 \mathrm{~dB}$, indicating good impedance matching. When we compare the search time for the case of $\rho=0 \mathrm{~cm}$, the search time is not significantly affected by $\rho$. The time to reach the final value is $<1.2 \mathrm{~s}$ using the three-step method. Using the one-step method, the time is approximately $3 \mathrm{~s}$ (worst case). Although the misalignment causes a shift in the estimated $Z_{\mathrm{IN}}$, we see that the proposed method can nevertheless find a good impedance match within a time period suitable for tracking dynamically moving coils.

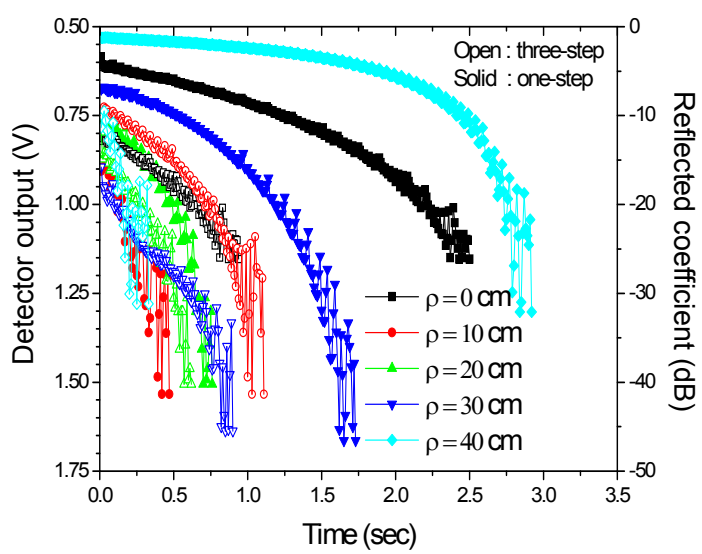

Figure 15. Measured detector output and reflection coefficient versus time for different center-to-center misalignments from $\rho=0 \mathrm{~cm}$ to $40 \mathrm{~cm}, d=15 \mathrm{~cm}$.

We measure efficiency when $\rho$ changes from $0 \mathrm{~cm}$ to $45 \mathrm{~cm}$ at two distances, as shown in Figure 16a for $d=15 \mathrm{~cm}$, and Figure $16 \mathrm{~b}$ for $d=30 \mathrm{~cm}$. At $d=15 \mathrm{~cm}$, the efficiency is rather sensitive to $\rho$ 
without matching. Using the proposed tuning method, relatively constant efficiency is obtained for up to $\rho=30 \mathrm{~cm}$. At $d=15 \mathrm{~cm}$, the measured efficiency improves by $39.1 \%$ and $35.1 \%$ at $\rho=10 \mathrm{~cm}$ and $\rho=40 \mathrm{~cm}$, respectively. At $d=30 \mathrm{~cm}$, the efficiencies with and without a tuning network both decrease with increasing $\rho$. Using the proposed method, the efficiency is significantly improved. At $\rho=35 \mathrm{~cm}$, it increases from $48.4 \%$ to $74.1 \%$. The simulated efficiencies are also shown, which agree well with the measured data.

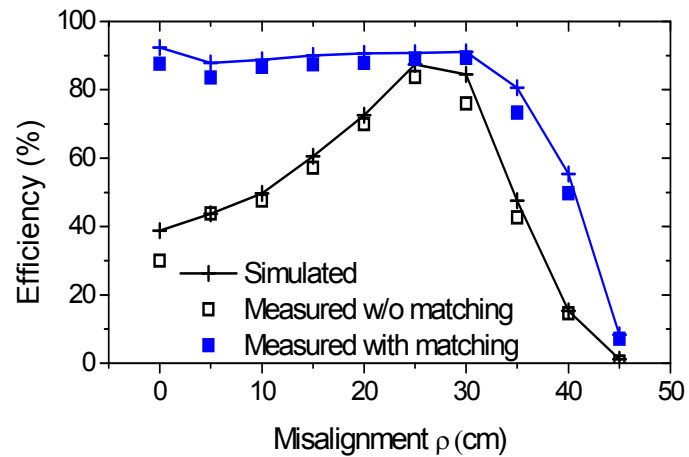

(a)

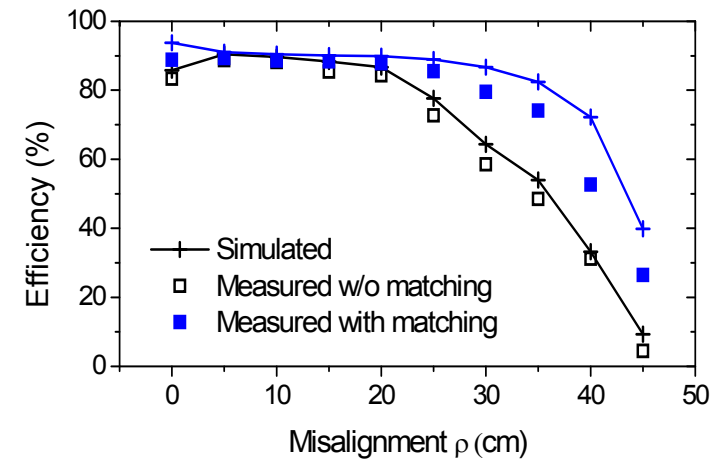

(b)

Figure 16. Efficiency comparison for different lateral misalignments at two distances:

(a) $d=15 \mathrm{~cm}$; and (b) $d=30 \mathrm{~cm}$.

\subsection{Angular Misalignment}

Figure 17 shows the measured trajectory of $Z_{\mathrm{IN} \text {,match }}$ when there are angular misalignments ranging from $\theta=0^{\circ}$ to $75^{\circ}$ in $15^{\circ}$ steps. The results show that $Z_{\mathrm{IN}, \text { match }}$ gradually converges to the center of the Smith chart using either method. At $\theta=30^{\circ}, \mid \Gamma_{\mathrm{IN}}$,match $\mid$ improves from $-14.1 \mathrm{~dB}$ to $-53.7 \mathrm{~dB}$ (and from $-2.8 \mathrm{~dB}$ to $-53.5 \mathrm{~dB}$ ) using the three-step method (and using the one-step method). Although the final $\left|\Gamma_{\mathrm{IN}, \text { match }}\right|$ values are similar, we achieve a significantly shorter trajectory of $Z_{\mathrm{IN}, \text { match }}$ using the three-step method. When $\theta$ changes from $0^{\circ}$ to $75^{\circ}$, the worst case of measured $\left|\Gamma_{\mathrm{IN} \text {,match }}\right|$ at the final point is $-31.8 \mathrm{~dB}$, indicating that the proposed system can handle significant angular misalignment.

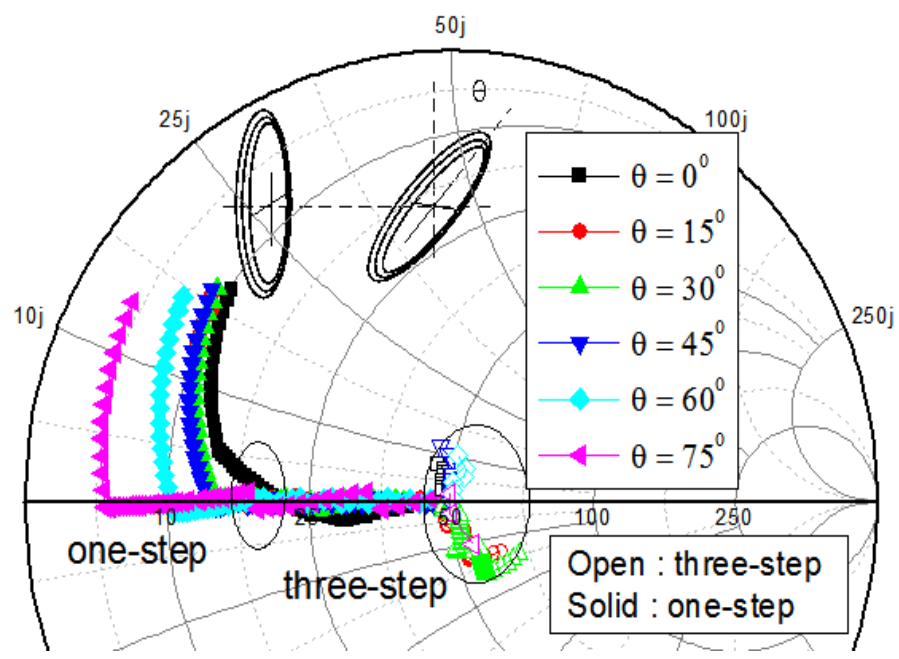

Figure 17. Trajectory of the measured input impedance for different angular misalignments from $\theta=0^{\circ}$ to $75^{\circ}$ in $15^{\circ}$ steps, $d=40 \mathrm{~cm}$. 
Figure 18 shows the measured $V_{\text {detect }}$ and $\mid \Gamma_{\mathrm{IN}}$,match $\mid$ as functions of time for different $\theta$. Using the three-step method, the maximum search time is approximately $1.7 \mathrm{~s}$, which occurs for $\theta=30^{\circ}$. In the range where $\theta$ changes from $0^{\circ}$ to $75^{\circ}$, the worst case $\mid \Gamma_{\mathrm{IN}}$,match $\mid$ is $-35 \mathrm{~dB}$. These results show that the proposed method can dynamically track and find a good impedance match under a wide range of angular misalignments.

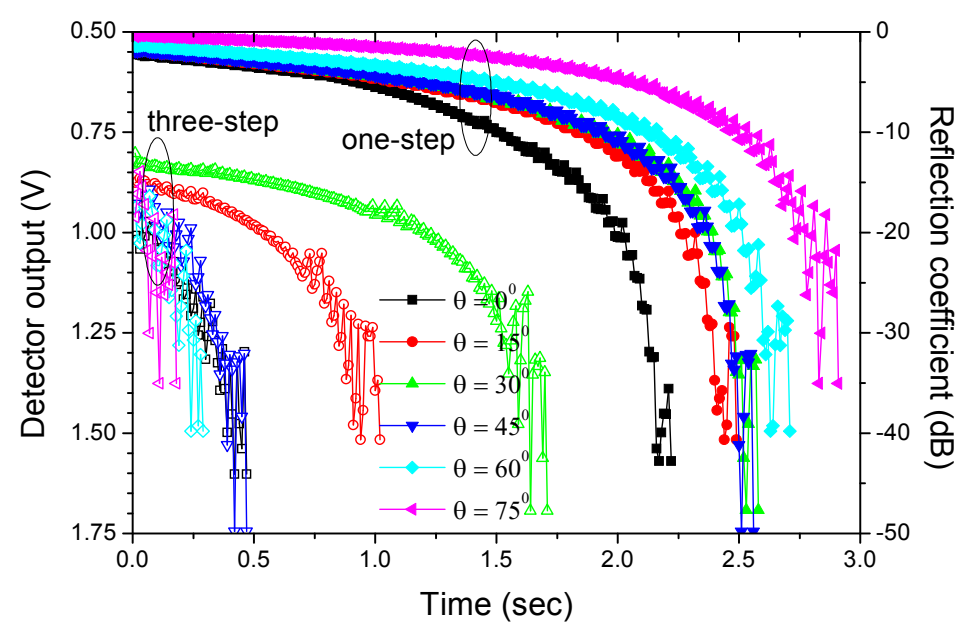

Figure 18. Measured detector output and reflection coefficient versus time for different angular misalignments, $d=40 \mathrm{~cm}$.

Figure 19 shows the measured efficiency when $\theta$ changes from $0^{\circ}$ to $75^{\circ}$ at $d=40 \mathrm{~cm}$. For up to $\theta=60^{\circ}$, the efficiency is higher than $74 \%$. For $\theta=75^{\circ}$, the efficiency improves from $26.6 \%$ to $58.2 \%$ using the proposed method. We note that the proposed system achieves an efficiency $>58 \%$ at $\theta=75^{\circ}$. Considering the high angle of rotation, this efficiency performance is notable. To verify the measured data, we use the coupling coefficients of Figure 4c. Using the method in [27], we obtain $k_{23}=0.029, k_{12}=k_{34}=0.29, k_{13}=k_{24}=0.013$, and $k_{14}=0.009$ at $\theta=75^{\circ}$. These numbers and the electrical parameters (Table 1) are used in the equivalent circuit model shown in Figure $1 b$. The simulated data show good agreement with the measured data. These results demonstrate excellent efficiency performance of the proposed system, which can deal with significant angular rotation.

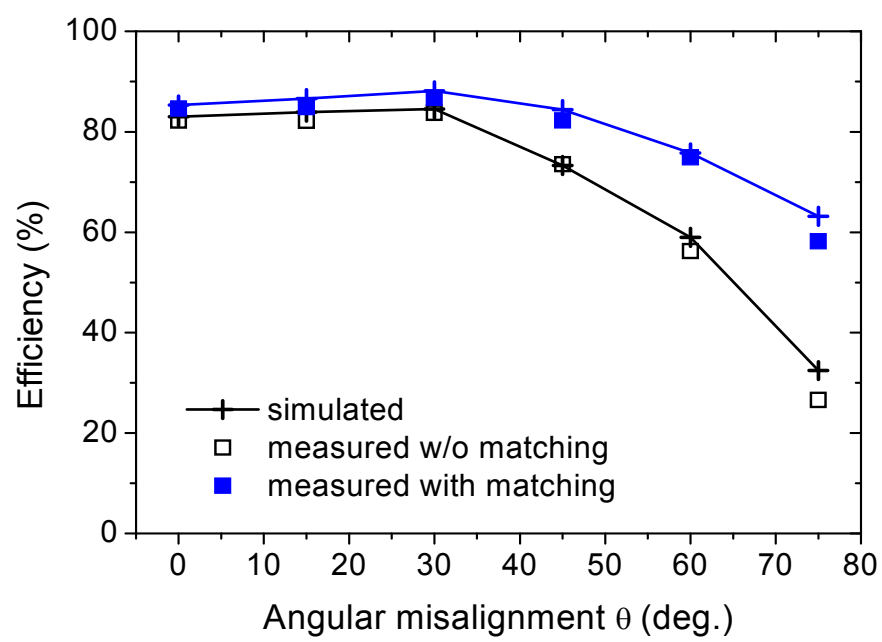

Figure 19. Efficiency comparison as a function of angular misalignment. 
To evaluate dynamic adaptation to unpredictable receiver position changes, we select a sequence of test cases, as shown in Figure 20. Each test case along the trajectory is indexed from 1 to 12, and it is built with a random combination of $d, \rho$, and $\theta$. Figure 21 a shows measured $Z_{\mathrm{IN}, \text { match }}$ and $\left|\Gamma_{\mathrm{IN}, \text { match }}\right|$ along the trajectory index. The maximum $\left|\Gamma_{\mathrm{IN}, \text { match }}\right|$ is $0.053(-25.5 \mathrm{~dB})$, which occurs at index-8 $\left(d=80 \mathrm{~cm}, \rho=20 \mathrm{~cm}\right.$, and $\left.\theta=15^{\circ}\right)$. Figure $21 \mathrm{~b}$ shows the measured efficiencies along the path. Among the selected test cases, the maximum efficiency is $90.5 \%$ at index 3 , while the minimum efficiency is $36.6 \%$ at index 6 . The highest efficiency improvement of $42.8 \%$ is achieved at index 7 . Overall, the proposed three-step method provides fast adaptation $(<1 \mathrm{~s})$ to changes of various receiver positions.

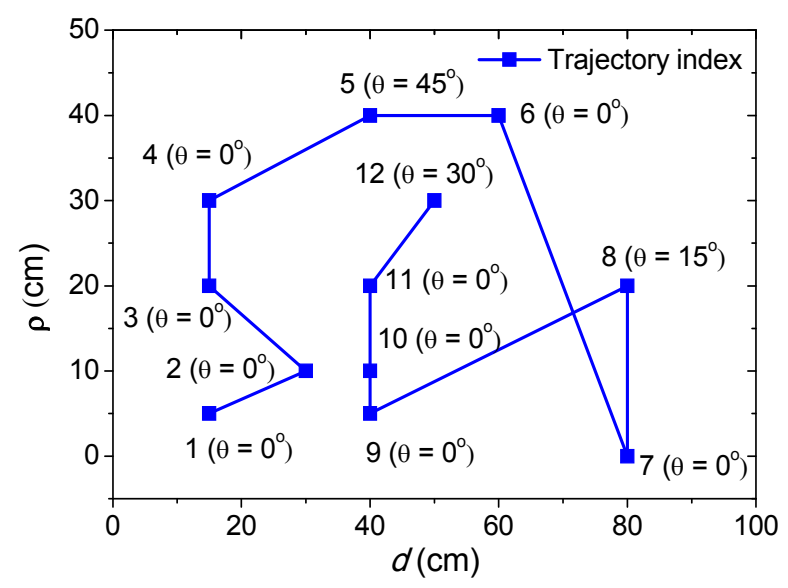

Figure 20. A sequence of test cases under changes of three geometric parameters.

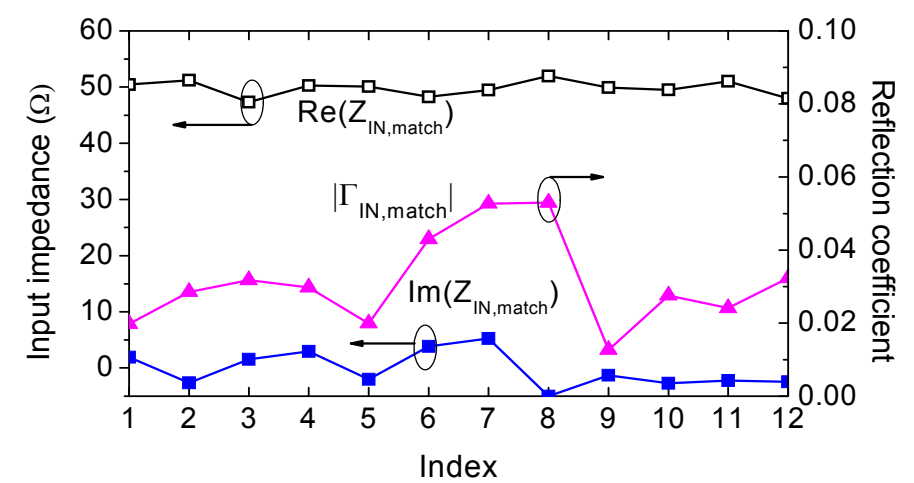

(a)

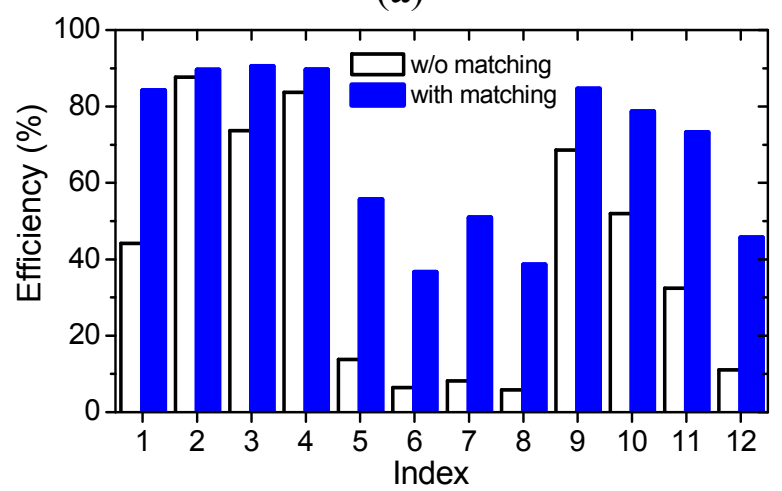

(b)

Figure 21. Measured results along the trajectory index: (a) input impedance and (b) efficiency. 
For practical application of a WPT system, characterization of leakage/fringe fields from WPT is important [36]. Therefore, we measure the leakage/fringe fields of the four-coil WPT without a ferrite core or aluminum shielding. A safety guideline for electromagnetic exposure is available from IEEE C95.1 standard [37]. The leakage magnetic B-field is measured at $5 \mathrm{~cm}$ from the edge of the resonator (35 cm from the center). We use a Narda 8715 survey meter equipped with an $8732 \mathrm{D}$ magnetic field probe. To find a worst-case high-power condition under which human safety is still secured, we increase $P_{\text {avail }}$ to $7.8 \mathrm{~W}$. At $d=100 \mathrm{~cm}$, the load coil receives about $2 \mathrm{~W}$. Under these conditions, the measured B-field is $1.34 \mathrm{~A} / \mathrm{m}$ at the side of resonator 2, gradually decreasing to $0.15 \mathrm{~A} / \mathrm{m}$ at the side of resonator 3. The IEEE C95.1 standard allows about $2 \mathrm{~A} / \mathrm{m}$ at $6.78 \mathrm{MHz}$. Under these operating conditions, therefore, the proposed WPT system can be used within the safety guidelines.

\section{Conclusions}

We propose an efficient three-step impedance searching method for a dynamically adaptable midrange WPT system. In the system calibration step, the coil parameters are obtained. In the coarse-search step, the coil position is estimated and the initial values for the matching network are determined. In the fine-search step, the impedance matching accuracy is improved by refining the values in the matching network. Measurements show that the proposed three-step method achieves good impedance matching within a short time and is therefore suitable for seamless wireless power operation. In addition, characterizations under several distance and alignment changes demonstrate the robustness of the proposed method. Using this method, the power transfer efficiency is significantly improved. In cases in which distance changed, we achieve a high efficiency of more than $50 \%$ for up to $d=80 \mathrm{~cm}$, with a maximum efficiency of $89.7 \%$. In the case of lateral misalignment, efficiency improved by $39.1 \%$ and $35.1 \%$ at $\rho=10 \mathrm{~cm}$ and $\rho=40 \mathrm{~cm}$, respectively, at $d=15 \mathrm{~cm}$. In the case of angular misalignment of $\theta=75^{\circ}$, the efficiency improved from $26.6 \%$ to $58.2 \%$ at $d=40 \mathrm{~cm}$. These results indicate that the proposed system can handle significant lateral and angular misalignment as well as distance changes. These results will be useful for convenient provision of power in dynamic environments in which mobile devices frequently change position. Moreover, further improvement in the dynamic adaptation is expected by adding communication capability between the receiver and the transmitter [14,15,38].

\section{Acknowledgments}

This research was supported by the Basic Science Research Program through the National Research Foundation of Korea (No. 2015R1A2A2A03004160).

\section{Author Contributions}

Thuc Phi Duong performed the simulations and measurements, and prepared initial paper draft. Jong-Wook Lee conceived the project, gave input to the theoretical work, organized and revised the paper.

\section{Conflicts of Interest}

The authors declare no conflict of interest. 


\section{References}

1. Hui, S.Y.; Zhong, W.X.; Lee, C.K. A critical review of recent progress in mid-range wireless power transfer. IEEE Trans. Power Electron. 2014, 29, 4500-4511.

2. Wireless Power Consortium. Available online: http://www.wirelesspowerconsortium.com (accessed on 12 November 2014).

3. Ahn, D.; Hong, S. A study on magnetic field repeater in wireless power transfer. IEEE Trans. Ind. Electron. 2013, 60, 360-371.

4. Rajagopalan, A.; RamRakhyani, A.K.; Schurig, D.; Lazzi, G. Improving power transfer efficiency of a short-range telemetry system using compact metamaterials. IEEE Trans. Microw. Theory Tech. 2014, 62, 947-955.

5. Ranaweera, A.L.A.K.; Duong, T.P.; Lee, J.W. Experimental investigation of compact metamaterial for high efficiency mid-range wireless power transfer applications. J. Appl. Phys. 2014, 116, doi:10.1063/1.4891715.

6. Kurs, A.; Karalis, A.; Moffatt, R.; Joannopoulos, J.D.; Fisher, P.; Soljacic, M. Wireless power transfer via strongly coupled magnetic resonances. Science 2007, 317, 83-86.

7. Chen, L.; Liu, S.; Zhou, Y.C.; Cui, T.J. An optimizable circuit structure for high-efficiency wireless power transfer. IEEE Trans. Ind. Electron. 2013, 60, 339-349.

8. Sample, A.P.; Meyer, D.A.; Smith, J.R. Analysis, experimental results, and range adaptation of magnetically coupled resonators for wireless power transfer. IEEE Trans. Ind. Electron. 2011, 58, 544-554.

9. Imura, T.; Hori, Y. Maximizing air gap and efficiency of magnetic resonant coupling for wireless power transfer using equivalent circuit and Neumann formula. IEEE Trans. Ind. Electron. 2011, $58,4746-4752$.

10. Zhong, W.; Lee, C.K.; Hui, S.Y.R. General analysis on the use of Tesla's resonators in domino forms for wireless power transfer. IEEE Trans. Ind. Electron. 2013, 60, 261-270.

11. Waffenschmidt, E. Wireless power for mobile devices. In Proceedings of the International Telecommunications Energy Conference, Amsterdam, The Netherlands, 9-13 October 2011; pp. 1-9.

12. Lee, S.G.; Hoang, H.; Choi, Y.H.; Bien, F. Efficiency improvement for magnetic resonance based wireless power transfer with axial-misalignment. Electron. Lett. 2012, 48, 339-340.

13. Park, J.; Tak, Y.; Kim, Y.; Kim, Y.; Nam, S. Investigation of adaptive matching methods for near-field wireless power transfer. IEEE Trans. Antennas Propag. 2011, 59, 1769-1773.

14. Kim, N.Y.; Kim, K.Y.; Choi, J.; Kim, C.-W. Adaptive frequency with power-level tracking system for efficient magnetic resonance wireless power transfer. Electron. Lett. 2012, 48, 452-454.

15. Sample, A.P.; Waters, B.H.; Wisdom, S.T.; Smith, J.R. Enabling seamless wireless power delivery in dynamic environments. Proc. IEEE 2013, 101, 1343-1358.

16. Waters, B.H.; Sample, A.P.; Smith, J.R. Adaptive impedance matching for magnetically coupled resonators. In Proceedings of the PIERS 2012, Moscow, Russia, 19-23 August 2012.

17. Beh, T.C.; Kato, M.; Imura, T.; Oh, S.; Hori, Y. Automated impedance matching system for robust wireless power via magnetic resonance coupling. IEEE Trans. Ind. Electron. 2013, 60, 3689-3698. 
18. Lim, Y.; Tang, H.; Lim, S.; Park, J. An adaptive impedance-matching network based on a novel capacitor matrix for wireless power transfer. IEEE Trans. Power Electron. 2014, 29, 4403-4413.

19. Yamakawa, M.; Shimamura, K.; Komurasaki, K.; Koizumi, H. Demonstration of automatic impedance-matching and constant power feeding to and electric helicopter via magnetic resonance coupling. Wirel. Eng. Technol. 2014, 5, 45-53.

20. Cannon, B.L.; Hoburg, J.F.; Stancil, D.D.; Goldstein, S.C. Magnetic resonant coupling as a potential means for wireless power transfer to multiple small receivers. IEEE Trans. Power Electron. 2009, 24, 1819-1825.

21. Ramo, S.; Whinnery, J.R.; Duzer, T.V. Fields and Waves in Communication Electronics, 3rd ed.; John Wiley \& Sons: Mississauga, ON, Canada, 1993.

22. Wheeler, H.A. Simple inductance formulas for radio coils. Proc. Inst. Radio Eng. 1928, 16, 1398-1400.

23. Akyel, C.; Babic, S.; Kinics, S. New and fast procedures for calculating the mutual inductance of coaxial circular coils. IEEE Trans. Magn. 2002, 38, 2367-2369.

24. Zierhofer, C.; Hochmair, E. Geometric approach for coupling enhancement of magnetically coupled coils. IEEE Trans. Biomed. Eng. 1996, 43, 708-714.

25. Grover, F.W. The calculation of the mutual inductance of circular filaments in any desired positions. Proc. IRE 1944, 32, 620-629.

26. Grover, F.W. Inductance Calculations; Dover Publications: Mineola, NY, USA, 1964.

27. Babic, S.; Sirois, F.; Akyel, C.; Girardi, C. Mutual inductance calculation between circular filaments arbitrarily positioned in space: Alternative to Grover's formula. IEEE Trans. Magn. 2010, 46, 3591-3600.

28. Hong, J.; Lancaster, M.J. Microstrip Filters for RF/Microwave Applications; John Wiley \& Sons: Mississauga, ON, Canada, 2001.

29. Jrad, A.; Perrier, A.-L.; Bourtoutian, R.; Dechamp, J.-M.; Ferrari, P. Design of an ultra compact electronically tunable microwave impedance transformer. Electron. Lett. 2005, 41, 707-709.

30. DS Relays_-Panasonic Industrial Devices DS2E. Available online: https://www3.panasonic.biz/ ac/e_download/control/relay (accessed on 6 May 2014).

31. Esram, T.; Chapman, P.L. Comparison of photovoltaic array maximum power point tracking techniques. IEEE Trans. Energy Convers. 2007, 22, 439-449.

32. RF Power Amplifier KMA2040. Available online: http://www.arww-modularrf.com/home modular_rf.cfm (accessed on 6 May 2014).

33. Directional Coupler MC05100-20. Available online: http://www.fairviewmicrowave.com/coaxial_ directional_couplers.htm (accessed on 6 May 2014).

34. Coaxial Power Detector ZX47-40+. Available online: http://www.minicircuits.com (accessed on 6 May 2014).

35. Duong, T.P.; Lee, J.-W. Experimental results of high-efficiency resonant coupling wireless power transfer using a variable coupling method. IEEE Microw. Wirel. Compon. Lett. 2011, 21, 442-444.

36. Christ, A.; Douglas, M.; Roman, J.; Cooper, E.; Sample, A.; Waters, B.; Smith, J.; Kuster, N. Evaluation of wireless resonant power transfer systems with human electromagnetic exposure limits. IEEE Trans. Electromagn. Compat. 2013, 55, 265-274. 
37. IEEE Std C95.1 IEEE Standard for Safety Levels With Respect to Human Exposure to Radio Frequency Electromagnetic Fields, $3 \mathrm{kHz}$ to $300 \mathrm{GHz}$; IEEE SCC28; IEEE Standards Department, International Committee on Electromagnetic Safety, the Institute of Electrical and Electronics Engineers: New York, NY, USA, 1999.

38. Moh, K.G.; Neri, F.; Moon, S.; Yeon, P.; Yu, J.; Cheon, Y.; Roh, Y.S.; Ko, M.; Park, B.H. A fully integrated $6 \mathrm{~W}$ wireless power receiver operating at $6.78 \mathrm{MHz}$ with magnetic resonance coupling, In IEEE International Solid State Circuits Conference, San Francisco, CA, USA, 1-4 February 2015; pp. 230-231.

(C) 2015 by the authors; licensee MDPI, Basel, Switzerland. This article is an open access article distributed under the terms and conditions of the Creative Commons Attribution license (http://creativecommons.org/licenses/by/4.0/). 